ARTICLE

DOI: $10.1038 / s 41467-017-01822-8$

\title{
Structure of Rap1b bound to talin reveals a pathway for triggering integrin activation
}

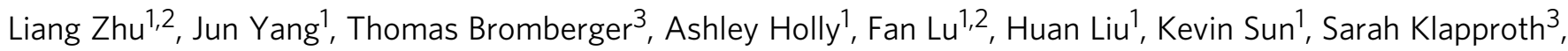
Jamila Hirbawi ${ }^{1}$, Tatiana V. Byzova1, Edward F. Plow ${ }^{1}$, Markus Moser ${ }^{3} \&$ Jun Qin $^{1,2}$

Activation of transmembrane receptor integrin by talin is essential for inducing cell adhesion. However, the pathway that recruits talin to the membrane, which critically controls talin's action, remains elusive. Membrane-anchored mammalian small GTPase Rap1 is known to bind talin-FO domain but the binding was shown to be weak and thus hardly studied. Here we show structurally that talin-F0 binds to human Rap1b like canonical Rap1 effectors despite little sequence homology, and disruption of the binding strongly impairs integrin activation, cell adhesion, and cell spreading. Furthermore, while being weak in conventional binary binding conditions, the Rap1b/talin interaction becomes strong upon attachment of activated Rap1b to vesicular membranes that mimic the agonist-induced microenvironment. These data identify a crucial Rap1-mediated membrane-targeting mechanism for talin to activate integrin. They further broadly caution the analyses of weak protein-protein interactions that may be pivotal for function but neglected in the absence of specific cellular microenvironments.

\footnotetext{
${ }^{1}$ Department of Molecular Cardiology, Lerner Research Institute, Cleveland Clinic, 9500 Euclid Avenue, Cleveland, OH 44195, USA. ${ }^{2}$ Department of Biochemistry, Case Western Reserve University, Cleveland, OH 44106, USA. ${ }^{3}$ Max-Planck-Institute of Biochemistry, Department of Molecular Medicine, 82152 Martinsried, Germany. Liang Zhu, Jun Yang and Thomas Bromberger contributed equally to this work. Correspondence and requests for materials should be addressed to M.M. (email: moser@biochem.mpg.de) or to J.Q. (email: qinj@ccf.org)
} 
$\mathrm{T}$ he adhesion of cells to the extracellular matrix (ECM) is essential for regulating a variety of physiological or pathological responses such as platelet aggregation, blood clotting, stroke, wound-healing, and cancer metastasis. Such adhesion critically depends upon integrins, a class of cell surface receptors that are $(\alpha / \beta)$ heterodimers with each subunit containing a small cytoplasmic tail (CT), a transmembrane segment, and a large extracellular domain. In unstimulated cells, integrin adopts an inactive conformation with low affinity for ECM ligands but, upon agonist stimulation, integrin $\mathrm{CT}$ is bound by intracellular proteins, in particular talin and/or kindlin, which trigger global conformational change of the extracellular domain to acquire high affinity for ECM ligand and initiate firm adhesion. This process, widely regarded as inside-out signaling or integrin activation, has been the central topic of cell adhesion research for nearly three decades ${ }^{1-6}$. Extensive efforts have been focused on investigating the mechanism of integrin activation by talin-a major cytoskeletal protein comprising an $\mathrm{N}$-terminal head domain (talin-H) and a C-terminal rod domain (talin-R). Talin-H contains four subdomains F0, F1, F2, and F3 in which F1-F3 constitutes a so-called FERM (4.1/ezrin/radixin/moesin)-like domain. Talin- $\mathrm{R}$ is made up of 13 consecutive helical bundles followed by a C-terminal dimerization domain (Fig. 1). Intact talin adopts an inactive conformation where the major integrinbinding site located in talin-H F3 domain is masked by talin- $\mathrm{R}$ (autoinhibition) $^{7-10}$. Talin may be unmasked via multiple mechanisms to bind integrin $\beta \mathrm{CT}^{7}, 8,11-13$ and trigger the receptor activation ${ }^{14-17}$.

While a great deal has been learned about talin, a fundamental issue still remains unresolved: how is cytoplasmic talin recruited to plasma membrane-a crucial step for talin to bind and activate integrin? A previous dogma, largely derived from studying prototypic integrin $\alpha_{\mathrm{II}} \beta_{3}$ from platelets, is that agonist stimulation activates small GTPase Rap1 on the membrane to recruit an effector called RIAM that in turn recruits $\operatorname{talin}^{5}{ }^{5} 18$. However, while both Rap1 and talin are highly abundant ${ }^{19}$, RIAM is present at very low levels in platelets with no other homologs detected ${ }^{20}$. More importantly, mice lacking RIAM are viable and the platelet functions including the $\alpha_{\mathrm{IIb}} \beta_{3}$ activation are totally normal ${ }^{20-22}$. By contrast, Rap1 and talin are both essential for integrin activation $^{4,5,18}$. For example, ablation of the Rap1 isoform Rap1b severely impairs $\alpha_{\mathrm{II}} \beta_{3}$ activation and platelet aggregation ${ }^{23}-$ defects that were also observed in talin knockout mice ${ }^{24,25}$. These observations strongly suggest a RIAM-independent engagement between Rap1 and talin to regulate integrin activation. Interestingly, previous studies reported a direct interaction between Rap1 and talin in mammals ${ }^{26}$ and in Dictyostelium ${ }^{27}$, but the affinity of the interaction was found to be low especially for that in mammals $\left(K_{\mathrm{d}} \sim 0.14 \mathrm{mM}\right)^{26}$. Since weak protein-protein interactions (PPIs) are typically assumed to be nonspecific and RIAM was widely regarded as a key bridge between Rap1 and talin, the Rap $1 /$ talin interaction was hardly studied at the mechanistic level. Through comprehensive structural, biochemical, and cell biological analyses, we discovered that talin is actually a distinct effector of Rap1 that recruits talin to membrane and triggers integrin activation. We further found that, while being weak in conventional binary binding assays, Rap1/talin interaction became strong when activated Rap1 was anchored to membrane that mimics the cellular condition. Our results thus uncover a mechanism of membrane-targeting of talin by Rap1 and also highlight the importance of weak PPIs that may be neglected in the absence of specific cellular microenvironments.

\section{Results}

Rap1 recognizes talin-F0 domain in a GTP-dependent manner. Rap1 is known to contain two highly homologous isoforms called Rapla and Rap1b ${ }^{28-31}$. In this study, we choose to focus on examining the interaction between the platelet-rich Rap $1 \mathrm{~b}$ and talin-F0. Like other small GTPases, Rap1b exists in inactive (GDP-bound) and active (GTP-bound) states. We first used a highly sensitive NMR technique called heteronuclear single quantum coherence (HSQC) ${ }^{32}$ to examine the Rap1b/talin-F0 interaction. Figure 2a and Supplementary Fig. 1a show that Rap1b loaded with GTP analog GMP-PNP induced residuespecific chemical shift changes of ${ }^{15} \mathrm{~N}$-talin-F0. Inactive Rap $1 \mathrm{~b}$ loaded with GDP also binds to talin-F0 but at weaker affinity as evidenced by smaller chemical shift changes than those with GMP-PNP (Fig. 2b). Thus, talin-F0 binds favorably to the active form of Raplb but, as shown in Fig. 2c, the binding affinity is quite low $\left(K_{\mathrm{d}} \sim 162 \mu \mathrm{M}\right)$ as previously concluded ${ }^{26}$. On the other hand, talin-F0 does not bind H-Ras (Supplementary Fig. 1b) that is near $90 \%$ homologous in terms of binding interface (overall similarity is $\sim 76 \%$ ) to Raplb (see also Supplementary Fig. 1c), suggesting that despite being weak, the talin-F0/Rap1b interaction is specific. To further investigate the specificity of Rap1b/talin-F0
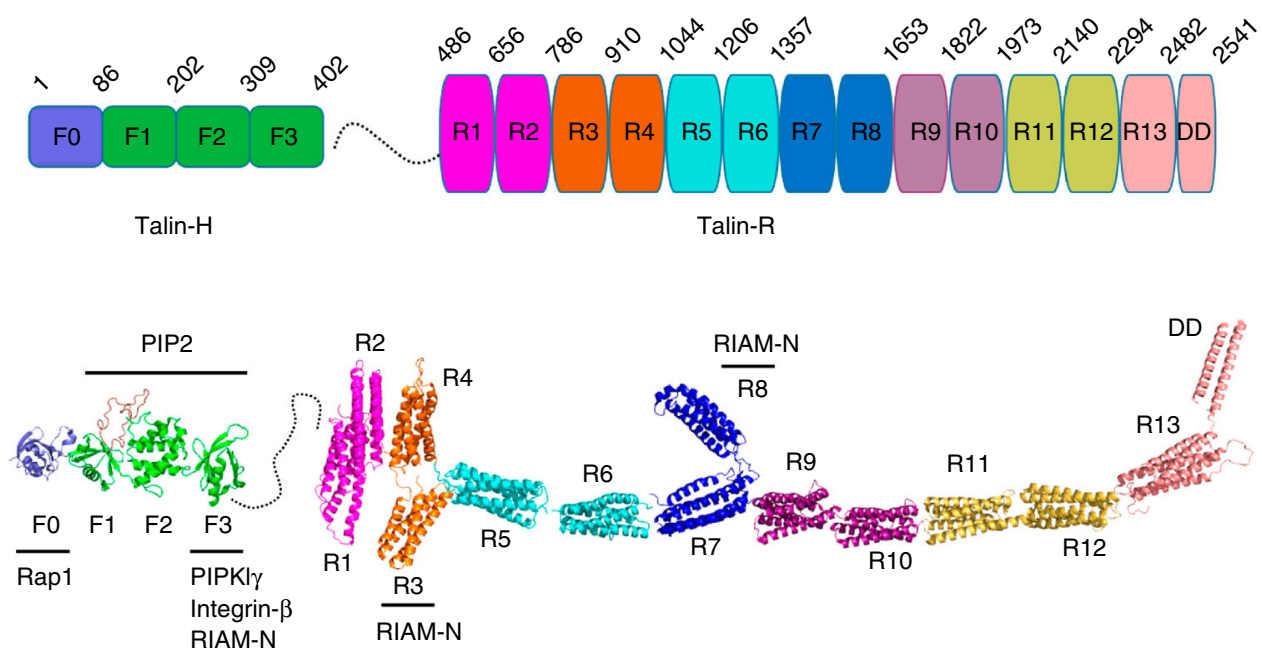

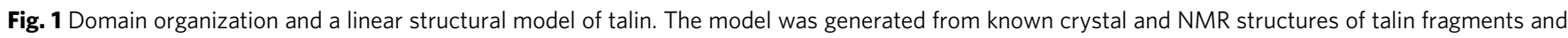
shown in cartoon representation. Critical talin-binding partners involved in talin membrane recruitment and activation are indicated in the figure. PIP2 phosphatidylinositol-4,5-bisphosphate, RIAM-N the $\mathrm{N}$ terminus of RIAM, PIPKI $\gamma$ type I phosphatidylinositol phosphate kinase isoform- $\gamma$ 


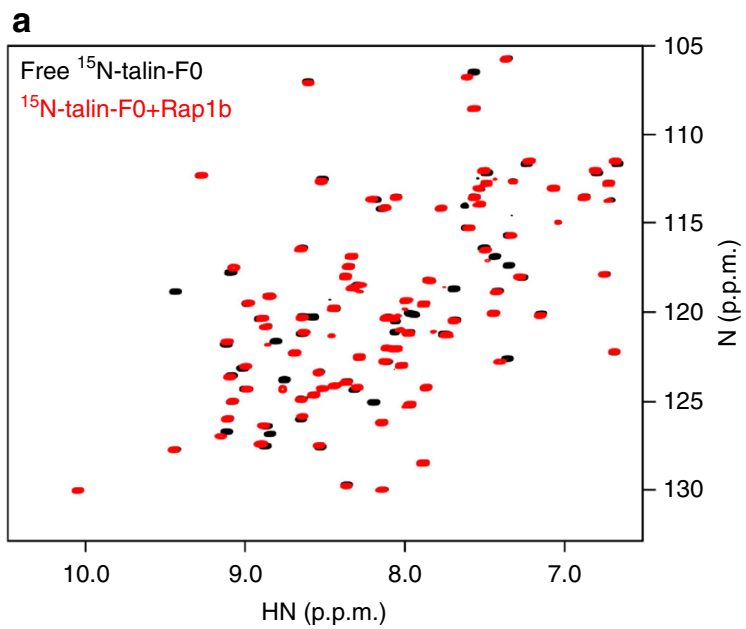

C

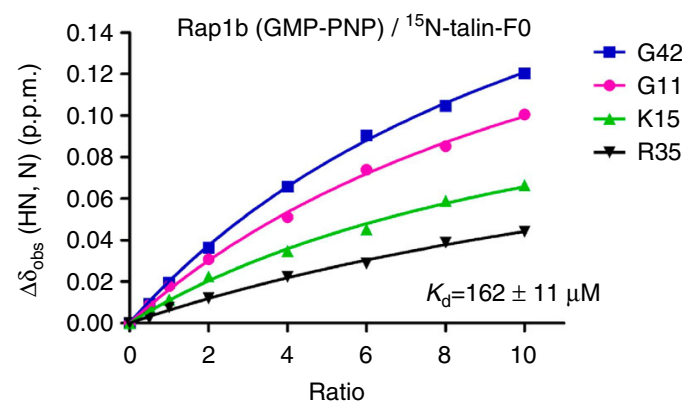

b

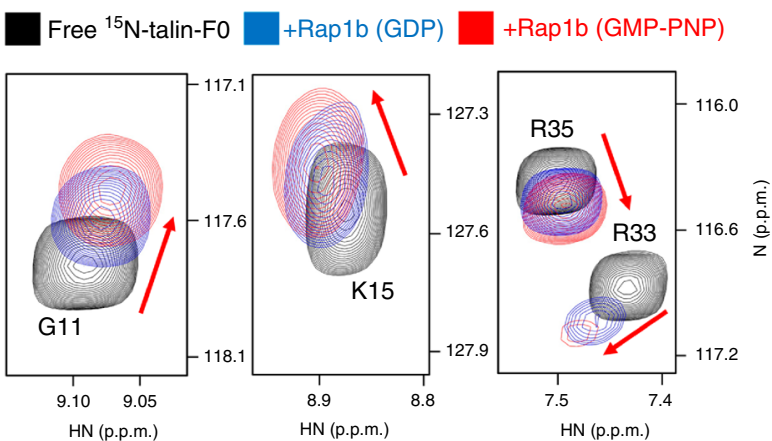

d

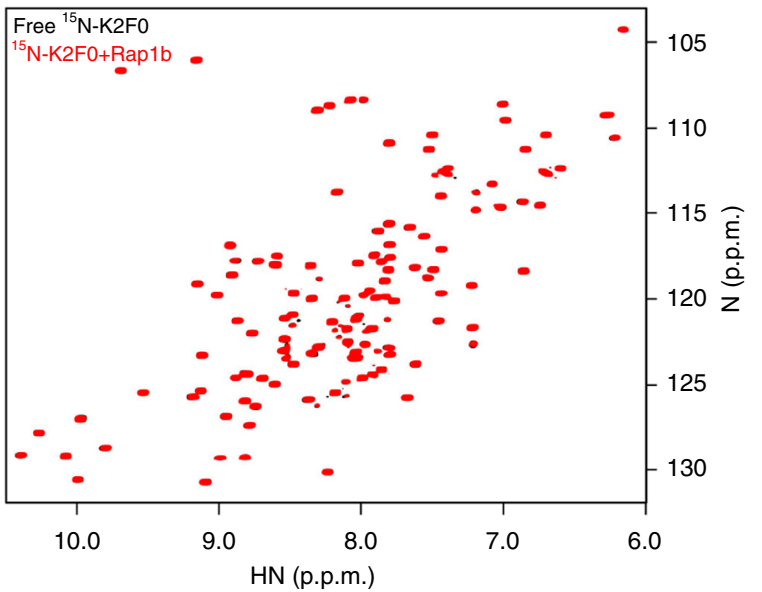

Fig. 2 Rap1b/talin-FO interaction is GTP-dependent and specific but is of weak affinity. a The HSQC spectra of $50 \mu \mathrm{M}{ }^{15} \mathrm{~N}$-labeled talin-FO in the absence (black) and presence of $125 \mu \mathrm{M}$ GMP-PNP loaded Rap1b (red). b The HSQC spectra (four representative residues were shown) of $50 \mu \mathrm{M}{ }^{15} \mathrm{~N}$-labeled talinFO in the absence (black) and presence of $125 \mu \mathrm{M}$ GDP loaded Rap1b (blue) or GMP-PNP loaded Rap1b (red). Note that Rap1b-GDP induced the same overall pattern of chemical shift changes of ${ }^{15} \mathrm{~N}$-labeled talin-FO as Rap1b-GMP-PNP does but with less peak shifts and broadenings, indicating a weaker affinity. c The affinity of Rap1b (GMP-PNP)/talin-FO interaction measured by HSQC titration. d The HSQC spectra of $50 \mu \mathrm{M}{ }^{15} \mathrm{~N}$-labeled kindlin2-FO in the absence (black) and presence of $125 \mu \mathrm{M}$ GMP-PNP loaded Rap1b (red)

interaction, we examined the Raplb binding to F0 domain of another integrin activator kindlin2 that is homologous to talin- $\mathrm{H}$ (Supplementary Fig. 2a) $)^{33}$, but no interaction was detected (Fig. 2d). Since talin-H contains FERM domain (F1-F3) prior to F0 and F1-F2 of KRIT1 FERM domain was shown to bind $\mathrm{Rap}^{1} \mathrm{~b}^{34}$, we also wondered whether F1-F2 of talin FERM would be involved in binding to Rap1b. However, Supplementary Fig. $2 \mathrm{~b}$ shows that Rap1b does not interact with talin FERM F1-F2. Overall, these data suggest that Rap1b engages with talin-F0 in a highly specific manner. Interestingly, NMR (Fig. 3a, Supplementary Figs. 1a and 2c) and Glutathione S-transferase (GST) pull-down (Fig. 3b) experiments revealed that active Rap1b also binds talin- $\mathrm{H}$ and full-length talin in the same manner as to talinF0. Given that full-length talin is autoinhibited with F0 being unmasked $^{7}$, these data suggest that active Rap1b specifically recognizes the F0 domain of resting talin.

Rap1b/talin interface exhibits distinct features. To understand how Rap1b recognizes talin, we decided to pursue the total structure of active human Rap1b $(\sim 20 \mathrm{kDa})$ in complex with talin-F0 $(\sim 10 \mathrm{kDa})$. Solution NMR is particularly suitable for structure determination of weak protein complexes ${ }^{32}$. By performing a series of three-dimensional (3D) heteronuclear experiments using a combination of ${ }^{1} \mathrm{H} /{ }^{15} \mathrm{~N} /{ }^{13} \mathrm{C}$-labeled and ${ }^{2} \mathrm{H} /{ }^{15} \mathrm{~N}$-labeled samples, we were able to obtain nearly complete resonance assignment, 3422 intramolecular NOEs, and 78 intermolecular NOEs (Supplementary Fig. 3), which allowed determination of the complex structure (Fig. 4a and Supplementary Table 1). Figure $4 \mathrm{~b}$ displays the cartoon diagram of the overall complex in which individual subunits, Rap1b and talin-F0, adopt a Ras family GTPase fold and an ubiquitin-like fold, respectively, as expected. The ubiquitin-like fold of talin-F0 has similarity to Ras-association (RA) domain present in known Rap1 effectors such as KRIT1, RIAM, and c-Raf1 (Fig. 4c). However, despite this similar fold, talin-F0 has little sequence homology with these Rap1b RAs (Fig. 4d) with only three positively charged residues being relatively conserved (highlighted in red in Fig. 4d). The detailed Rap1b/talin-F0 interface is summarized in Fig. 4e and Supplementary Fig. 4a and compared with other Rap1b effectors (Supplementary Table 2). Majority of the contacts in the Rap1b/talin-F0 complex are different from other Rap1b/effector complexes (Supplementary Table 2) apparently due to the scant sequence homology of talin-F0 with other Rap1b effectors (Fig. 4d). A particularly distinct feature in the Raplb/talin-F0 complex is the existence of a hydrophobic core between I36/P37 of talin-F0 and V21/I27/V29 of Rap1b (Fig. 4e and Supplementary Fig. 4b). Such core was not observed in other Raplb/effector complexes. To understand how Raplb but not the highly homologous H-Ras binds talin-F0 (Supplementary Fig. 1b), we mutated I27 and K31 of Rap1b, which closely contact with talinF0, into corresponding H-Ras residues, Histidine and Glutamate, respectively. Both mutations showed reduced binding to talin-F0 (Supplementary Fig. 4c). By contrast, both Rap1b and H-Ras bind equally well to RIAM as shown before ${ }^{35}$ as also confirmed in 
a

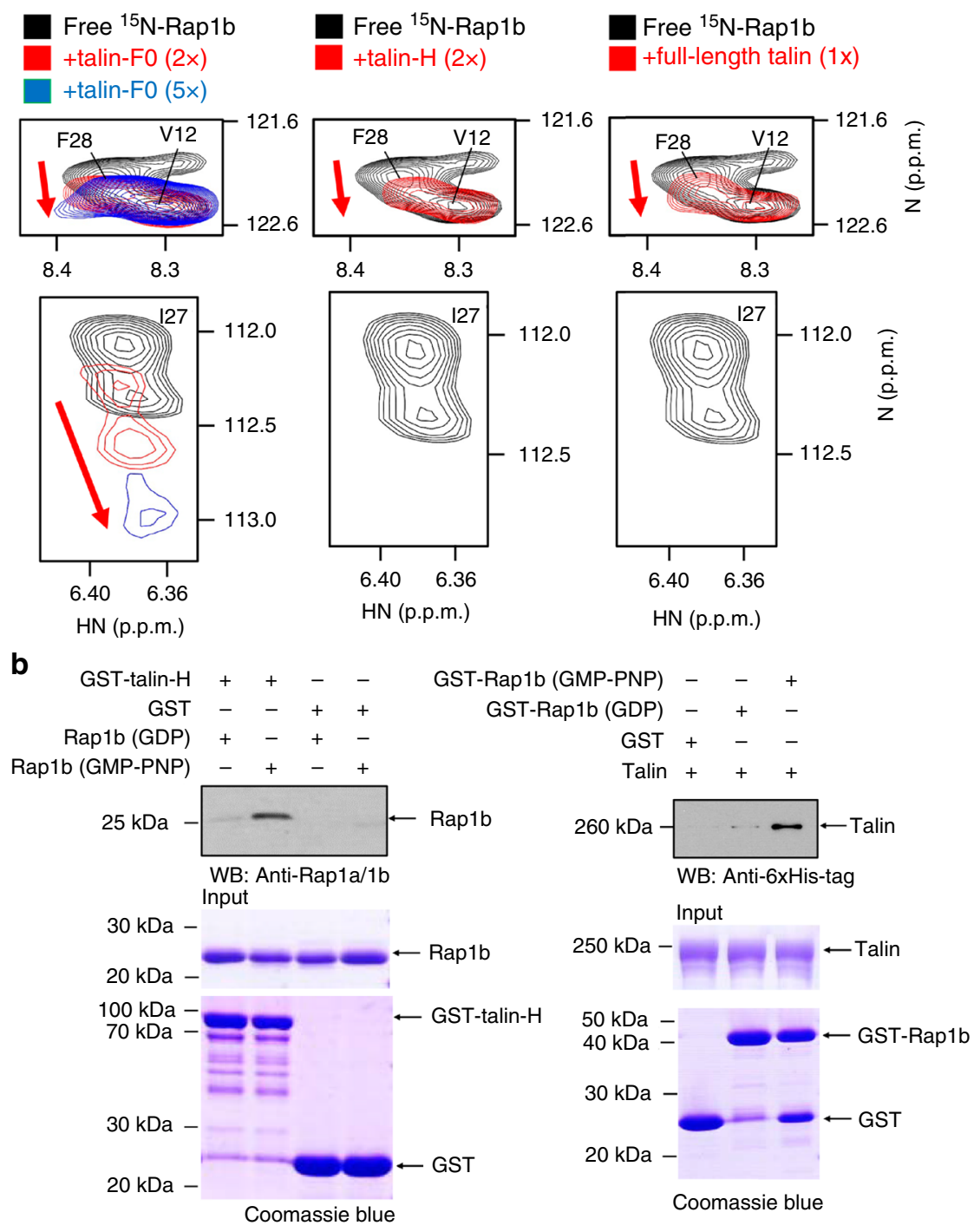

Fig. 3 Rap1b recognizes FO domain of talin in a GTP-dependent manner. a The HSQC spectra (representative regions were shown) of $45 \mu M$ GMP-PNP loaded ${ }^{15} \mathrm{~N}$-labeled Rap1b (1-167) in the absence (black) and presence of $90 \mu \mathrm{M}$ talin-FO (red), $225 \mu \mathrm{M}$ talin-FO (blue), $90 \mu \mathrm{M}$ talin-H (red), or $45 \mu \mathrm{M}$ fulllength talin (red). Note that the peak of $\mathrm{I} 27$ was broadened in the presence of talin-H or full-length talin. $\mathbf{b}$ GST pull-down assays to show that Rap1b interacts with both talin-H and full-length talin in a GTP-dependent manner. Full blot/gel images are shown in Supplementary Figs. 11 and 12

Supplementary Fig. 4d and Supplementary Fig. 16. These data provide insight into the high specificity of the talin binding to Raplb despite the weaker affinity than that between talin and RIAM (Supplementary Fig. 4e).

Further analysis revealed that, while the Rap1b/talin-F0 interface exhibits distinct features (Fig. 4e, Supplementary Fig. 4a, and Supplementary Table 2), the overall topology of the complex shares similarity with other Rap1b/effector complexes (Fig. 4b, c). A key determinant for this similarity appears to be the antiparallel $\beta$-sheet between Rap1b $\beta 2$ strand and talin-F0 $\beta 2$ strand (Fig. $4 \mathrm{~b}$ and Supplementary Fig. 4a), as also present in other Rap1b/ effector complexes (Fig. 4c). In addition, charge-charge interactions in the talin-F0/Rap1b complex, which involve talin-F0 K7 with Rap1b E37 and talin-F0 K15/R35 with Rap1b D33, are relatively conserved in other Raplb/effector complexes (Fig. 4d and Supplementary Table 2). By contrast, kindlin2-F0, which is not a Rap1b effector (Fig. 2d) but is highly homologous to talinF0 (Supplementary Fig. 2a), lacks two positively charged residues in the corresponding positions (Fig. 4d).
In summary, the Rap1b/talin-F0 interface is quite unique yet resembles other Raplb/effector complexes in overall topology. These structural and binding data suggest that talin is a direct effector of Rap1. Interestingly, the interface residues are highly conserved in both talin/Rap1 isoforms and in different species (Supplementary Fig. 5a, b), suggesting that Rap1/talin recognition is evolutionally conserved.

Raplb binding to talin is crucial for integrin activation. To further evaluate the importance of the Rap1b/talin interaction, we performed the structure-based analysis and mutated two critical interface residues K15 and R35 of talin-F0 (Fig. 4e and Supplementary Fig. 4a) into alanines. Figure $5 \mathrm{a}$ and Supplementary Fig. 6 show that K15A/R35A double mutations (DM) drastically reduced the Rap1b interaction to talin-F0 as well as talin-H. Since talin- $\mathrm{H}$ is the unit responsible for binding and activating integ$\operatorname{rin}^{5,36}$, we next tested the impact of the Raplb-binding defective mutations on its ability to activate $\alpha_{\mathrm{IIb}} \beta_{3}$-the prototypic integrin widely used to study integrin activation. To this end, we 
a

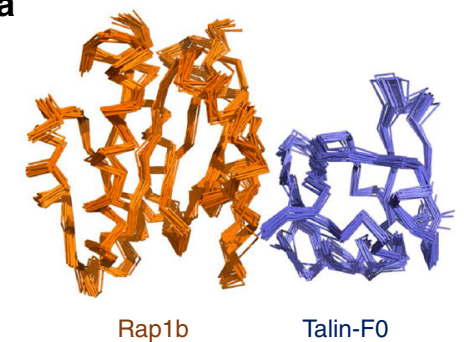

b

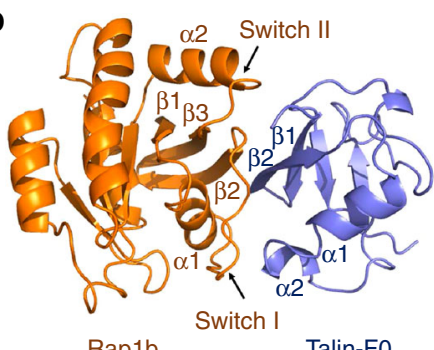

Rap1b

C

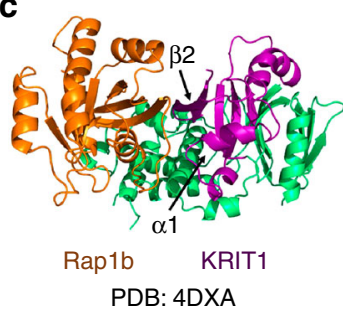

PDB: 4DXA

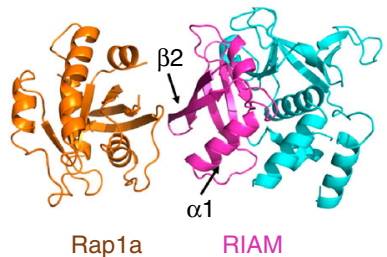

PDB: 4KVG

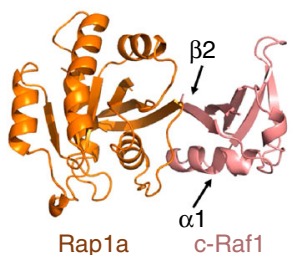

PDB: 1GUA

d
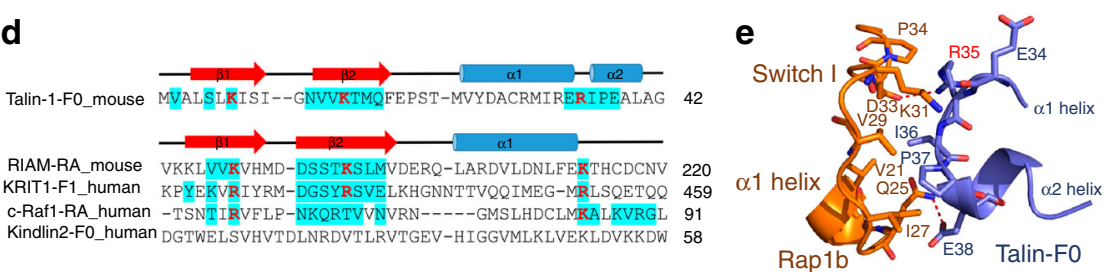

Fig. 4 Solution structure of Rap1b/talin-FO complex by NMR. a Superposition of 20 calculated Rap1b/talin-FO complex structures with lowest energies (shown in ribbon representation). b Cartoon representation of the Rap1b/talin-FO complex structure with the lowest energy. c Current solved complex structures of Rap1 and its effector proteins (shown in cartoon representation). d Structure-based sequence alignment of talin-FO and RA domain containing Rap1b effector proteins or kindlin2-FO (only binding interfaces were shown). Residues involved in the binding interface with a cutoff of $4 \AA$ are highlighted in cyan. Conserved residues are colored in red. e Detailed interaction diagram between the $\alpha 1$ helix and switch I of Rap1b and the $\alpha 2$ helix of talin-F0. Hydrogen bond or salt bridge is represented by red dashed line

transfected wild-type (WT) talin-H or talin-H DM into Chinese hamster ovary (CHO) A5 cells stably expressing $\alpha_{\text {IIb }} \beta_{3}$ (Supplementary Fig. 7a). Figure 5b shows that, as compared to the WT talin- $\mathrm{H}$, the talin- $\mathrm{H}$ mutant-induced $\alpha_{\mathrm{II}} \beta_{3}$ activation was substantially reduced. These results provide strong evidence that the interaction of talin-H with Rap1b is crucial for the talin-Hmediated integrin activation. They also explain a previously reported phenomenon where deletion of F0 domain in talin- $\mathrm{H}$ significantly impaired the integrin activation ${ }^{37}$. To further demonstrate that the impaired interaction between talin DM and Rap1 has an impact on integrin-mediated functions, we retrovirally transduced talin-null fibroblasts $\left(\operatorname{talin}^{1 / 2 \mathrm{dko}}\right.$ ), which show a strong defect in cell adhesion and spreading ${ }^{38}$, with $\mathrm{C}$ terminally ypet-tagged talin WT as well as talin DM. The transduced cells were FACS-sorted for equal talin and talin DM expression as confirmed by flow cytometry and western blotting (Supplementary Figs. 7b and 17). Re-expression of both WT talin and talin DM induced cell spreading of talin ${ }^{1 / 2 \mathrm{dko}}$ cells with WT and mutant talin localizing to paxillin-positive focal adhesions (FAs; Supplementary Figs. 7c, d). We then performed adhesion assays on various integrin ligands and observed that talin DM only partially rescued cell adhesion of talin ${ }^{1 / 2 \mathrm{dko}}$ cells to fibronectin (FN), laminin-111 (LN), and vitronectin (VN) compared to WT talin, whereas integrin-independent adhesion to poly-L-lysine was unchanged (Fig. 5c). As expected, talin ${ }^{1 / 2 \mathrm{dko}}$ cells expressing ypet as a control hardly adhered to any integrin ligand (Fig. 5c). Importantly, expression of mutant talin did not alter the expression levels of surface integrins nor those of RIAM and Rap1 (Supplementary Figs. 7b, e). Next, we plated the cells on FN and measured cell spreading for $4 \mathrm{~h}$. While ypet-transduced talin ${ }^{1 / 2 \mathrm{dko}}$ cells remained roundish and did not spread, talin DM cells displayed a significant spreading defect compared to talin WTtransduced cells (Fig. 5d).

We hypothesize that the reduced PAC1 binding, cell adhesion, and spreading of talin DM-expressing cells are due to impaired integrin activation caused by deficient talin recruitment to the integrin site at the plasma membrane. To test this hypothesis experimentally, we seeded WT talin and talin DM-expressing cells on FN-coated micropatterns for $4 \mathrm{~h}$ and measured the number and size of paxillin-positive FAs. This analysis reveals that indeed the FA area and number per cell are significantly reduced in cells expressing talin DM compared to those expressing WT talin (Figs. 5e-g). The mean FA size (Fig. 5h) was also reduced by talin DM, although with a $p$ value of 0.09 . To further test whether impaired talin/Rap1 interaction affects the recruitment of talin DM to FAs, we measured the intensity of ypet fluorescence within FAs and correlated it to the total cellular ypet fluorescence intensity. We observed a significantly reduced relative intensity of ypet signal within FAs in talin DMtransduced cells, which suggests that the talin/Rap1b interaction is important for talin recruitment to FAs (Fig. 5i). Collectively, our data provide strong evidence for the physiological importance of direct Rap1/talin interaction in integrin regulation.

Membrane-anchored Rap1b shows enhanced binding to talin. While the forgoing data demonstrated the unique specificity of Rap1b/talin interaction and its importance in regulating integrinmediated functions, a key issue still remains: how can such interaction with $\sim 0.16 \mathrm{mM}$ affinity be effective since protein concentration in cells is typically much lower than $0.16 \mathrm{mM}$ ? We attempted to address this issue from two angles. First, both Rap1 
and talin are highly abundant in cells. For example, recent proteomic studies showed that Raplb and talin belong to the top most abundant proteins in platelets with copy numbers of $\sim 200,000$, which are nearly $1 / 4$ of the most abundant platelet protein actin $^{19}$. The high levels of Rap1 and talin in platelets are consistent with our protein expression analysis (Fig. 6a). Given that the average concentration of actin is above $200 \mu \mathrm{M}$ in platelets $^{39}$, the estimated concentration of Rap1b or talin in platelets should be at least $50 \mu \mathrm{M}$. By contrast, the RIAM level is very low in both human and murine platelets (Fig. 6a), which was also confirmed by quantitative mass spectrometry with a copy number of only $162^{19}$. Thus, the high concentrations of Rap 1 and talin increase the chance for their direct interaction to occur. Second, given that Ras family GTPases including Rap1 are all

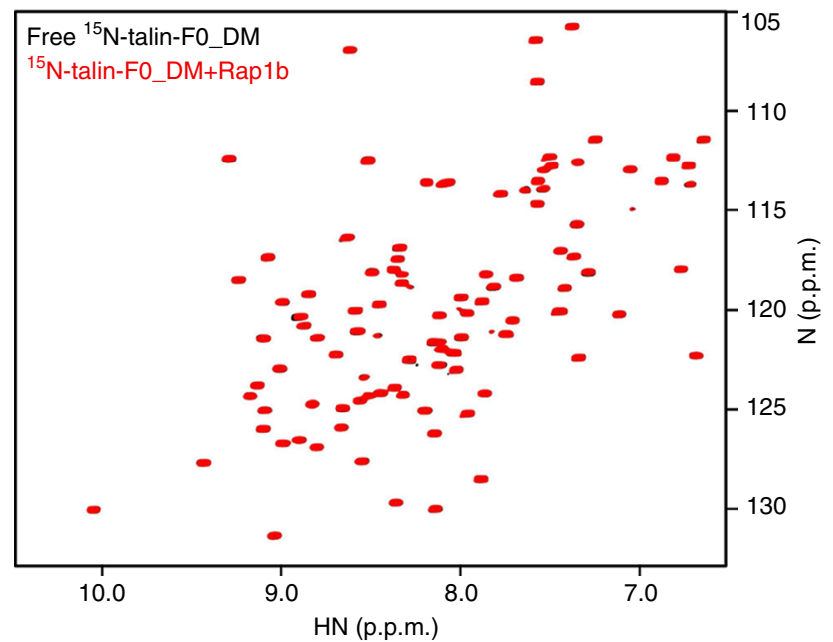

b

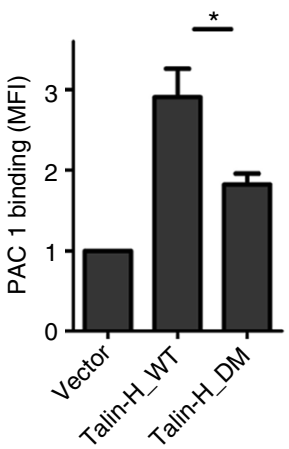

C
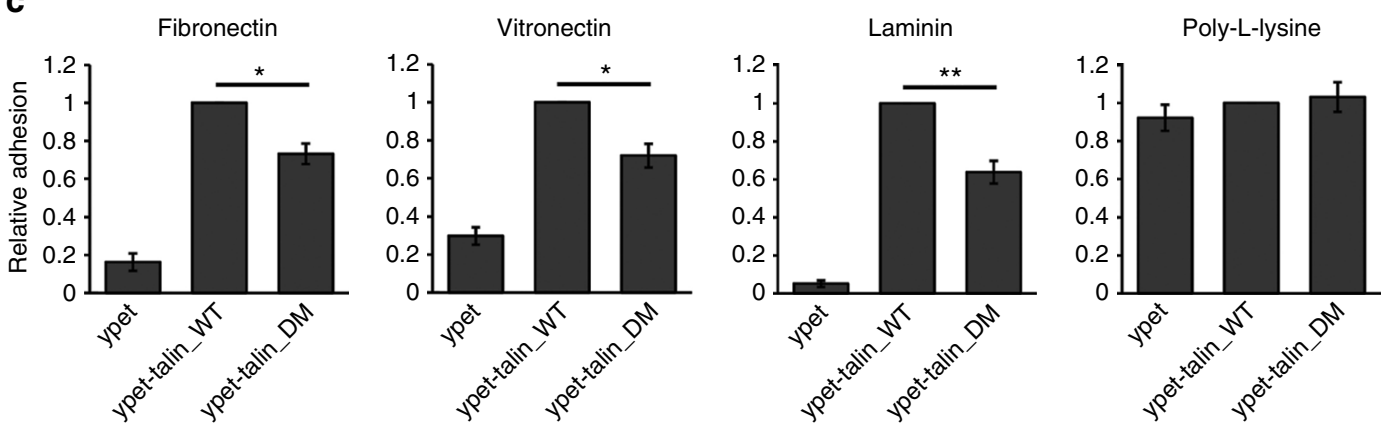

d

$\mathbf{e}$ $\multimap$-ypet $\prec$ ypet-talin_WT $\_$-ypet-talin_DM

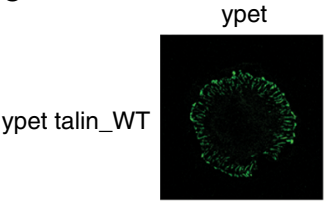

Paxillin
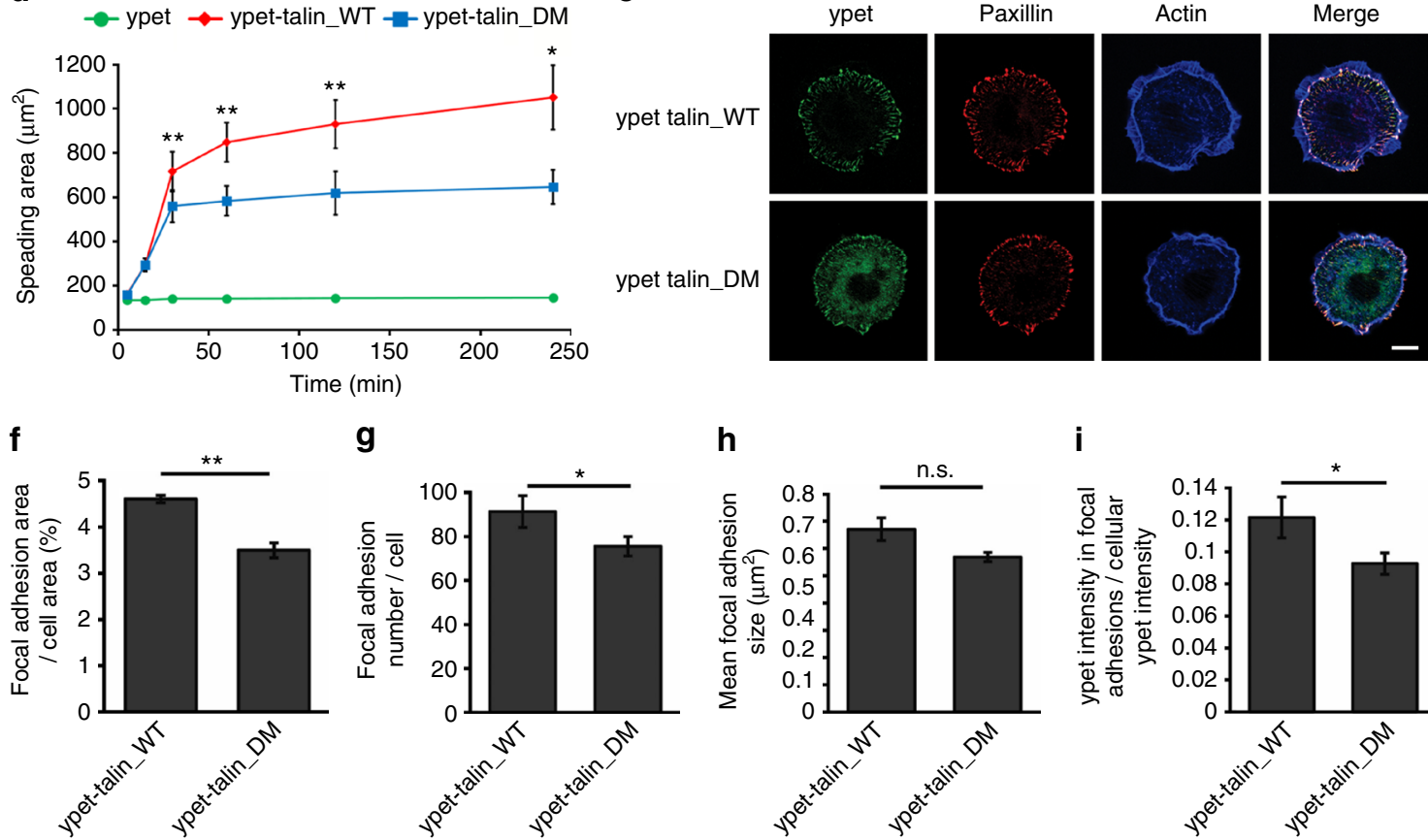

g

h
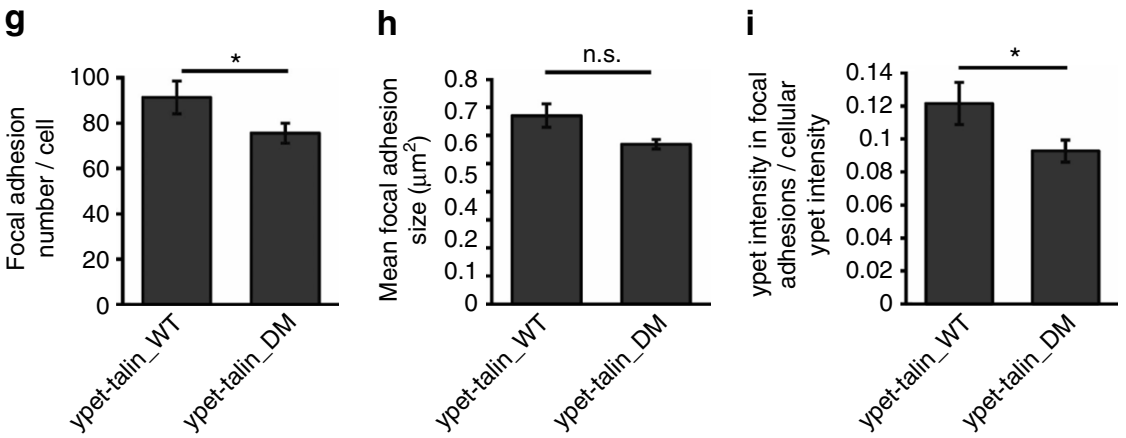
known to be attached to membrane via Cys prenylation of their C-terminal CAAX motif ${ }^{29}$ and talin- $\mathrm{H}$ also binds to membrane via a large positively charged surface on F1, F2, and F3 domains 8 , 40 , we reasoned that Rap1 and talin- $\mathrm{H}$ might be more tightly associated if Rap1 were prenylated with membrane to mimic the true cellular microenvironment (Fig. 6b). Our Rap1b/talinF0 structure superimposed with the structure of talin-H supports this possibility in that the CAAX containing $\mathrm{C}$ terminus of Raplb and the positively charged surface of talin- $\mathrm{H}$ align well to all face toward the membrane surface (Fig. 6b). To address this possibility experimentally, we prepared membrane vesicles with anchored Rap1b using the well-established thiol-maleimide crosslinking method $^{41}$ (Supplementary Fig. 8a). As shown in Fig. 6c, membrane-anchored Raplb indeed bound much more robustly to talin- $\mathrm{H}$ than Rap $1 \mathrm{~b}$ unanchored to membrane. Isothermal titration calorimetry (ITC) experiments revealed a $K_{\mathrm{d}}$ of $\sim 1.52$ $\mu \mathrm{M}$ for membrane-anchored active Raplb to bind to talin- $\mathrm{H}$ (Supplementary Fig. 8b), which indicates a more than two orders of magnitude stronger binding than that measured without membrane (Fig. 2c and Supplementary Fig. 4e). Consistently, the membrane-binding capacity of talin- $\mathrm{H}$ was also substantially enhanced by Rap1 anchored to the membrane, but such enhancement was diminished for talin-H DM that is defective for binding to Rap1 (Fig. 6d). Importantly, the binding of membrane-anchored Rap1b to talin-H is GTP-dependent as shown by two independent methods: GST pull-down assay (Supplementary Figs. 8c and 18) and co-sedimentation assay (Supplementary Figs. 9a and 19). Such GTP dependence indicates that the effective membrane targeting of talin requires active Raplb. These findings provide key evidence for talin being a bona fide Rap1 effector and suggest how agonist-activated and membrane-anchored Rap1 can effectively recruit talin to the membrane surface. Although full-length talin binds to membrane more weakly than talin- $\mathrm{H}$ possibly due to some degree of autoinhibition for the former, membrane with anchored Rap1b clearly binds more potently to full-length talin contrasting to the scenario where Raplb was not attached to the membrane (Fig. 6e). Similar to membrane-mediated Rap1b/talin-H binding (Supplementary Fig. 9a), membrane-anchored Rap1b also interacts with full-length talin in a GTP-dependent manner (Supplementary Fig. 9b). Importantly, since full-length talin adopts an autoinhibited conformation ${ }^{7}, 10$, which may be unmasked through a pull-push mechanism by binding to the membrane ${ }^{8}$, our data further suggest that Rap1 not only promotes the membrane targeting but also leads to activation of talin via this mechanism (Fig. 6b). In order to test this hypothesis, we performed NMRbased competition experiments by examining the interaction of ${ }^{15} \mathrm{~N} /{ }^{2} \mathrm{H}$-labeled inhibitory talin rod domain 9 (talin-R9) with talin- $\mathrm{H}$ in the absence and presence of membrane-anchored Rap1b. Supplementary Fig. 9c shows that, while talin- $\mathrm{H}$ induces residue-specific chemical shift changes of talin-R9 as the signature of the autoinhibition ${ }^{8}$, such chemical shift changes were suppressed by membrane-anchored Rap1b, thus demonstrating the talin-unmasking process.

\section{Discussion}

Rap1 was discovered nearly three decades ago ${ }^{42}$, and its two isoforms Rap1a and Rap1b (with $~ 95 \%$ identity, see also Supplementary Fig. 5b) are regarded as the key membrane-associated small GTPases to target proteins to the plasma membrane to regulate diverse cellular responses ${ }^{28-31}$. A large body of data have indicated that Rap1 is critically involved in activation of integrins $^{5}, 18,43$, but the immediate downstream effector of Rap1 has been highly elusive. Our studies have now obtained an important clue on this longstanding mystery. We found that the activated Rap 1b interacts with the F0 domain of talin-the key mediator of integrin activation, and we determined the NMR structure of the Rap1b/talin-F0 complex. Despite little sequence homology between talin-F0 and other Rap1 effectors, the overall topology of the complex was found to resemble those known Rap1/effector complexes. Moreover, disruption of the Rap1b/talin interface substantially impaired the integrin $\alpha_{\mathrm{IIb}} \beta_{3}$ activation, cell adhesion, and cell spreading. Interestingly, we found that, while being weak measured in conventional binary conditions ${ }^{26}$, the Rap $1 \mathrm{~b} /$ talin interaction became quite strong when Raplb was anchored to membrane-a condition mimicking the cellular microenvironment of Ras family GTPases ${ }^{41}$ and also favoring talin unmasking as indicated previously ${ }^{8}$. Overall, our data suggest a crucial pathway by which agonist-activated Rapl directly promotes the membrane recruitment of talin, which further leads to the talin unmasking and integrin activation (Fig. 6b). Our data also provide an explanation of why Rapl and talin are essential for integrin activation, whereas RIAM is dispensable for activation of most integrins expressed on many cell types as indicated by the recent series of genetic studies ${ }^{20-22}$. On the other hand, RIAM, which is highly abundant in leukocytes, was shown to play an important role in regulating leukocyte integrin function ${ }^{21,22}$ by possibly forming the so-called "MIT complex" with talin and integrin $^{44}$, indicating that the direct Rap1/talin pathway plays a minor role in regulating the leukocyte $\beta_{2}$ integrins. The fact that talin DM still partially supports cell adhesion and spreading of $\operatorname{talin}^{1 / 2 \mathrm{dko}}$ cells (Fig. 5c, d) further indicates the existence of other compensatory pathways including "Rap1-RIAM-talin" and "PIPKI $\gamma /$ PIP2-talin"7, 8, 11 . More detailed investigations are needed to understand how these different pathways are turned on or cooperated in a spatiotemporal manner. Nevertheless, our data, combined with the genetic evidence of Rap1 and talin in integrin activation, suggest that direct Rap1-talin interaction critically regulates integrin activity.

In addition to uncovering the physiological role of the Rap1b/ talin interaction, our findings also bear broad implications in the analyses of PPIs. Tens of thousands of PPIs in cells form a web of intricate communication networks for regulating diverse

\footnotetext{
Fig. 5 The Rap1b binding to talin is crucial for integrin activation. a The HSQC spectra of $50 \mu \mathrm{M}{ }^{15} \mathrm{~N}$-labeled talin-FO_DM (K15A, R35A) in the absence (black) and presence of $125 \mu \mathrm{M}$ GMP-PNP loaded Rap1b (red). b Integrin activation assay in CHO A5 cells, which stably express integrin $\alpha_{\| 1} \beta_{3}$. Double mutations (K15A, R35A) in talin-H substantially decreases integrin activation. The data are shown as means \pm S.E.M. from four independent experiments. ${ }^{\star} p<0.05$. c Static adhesion of talin ${ }^{1 / 2 \mathrm{dko}}$ fibroblasts, expressing either ypet alone, ypet-tagged talin WT, or ypet-tagged talin DM (K15A, R35A). Number of adherent cells was quantified by measuring absorbance of crystal violet staining. Values measured for talin WT-transduced cells were set to one in each of six independent experiments. Values are given as mean \pm S.E.M. ${ }^{\star} p<0.05$ and ${ }^{\star \star} p<0.01$. d Spreading of talin ${ }^{1 / 2 d k o}$ fibroblasts expressing ypet (green), ypet-tagged talin WT (red), or ypet-tagged talin DM (blue) on a fibronectin-coated surface, measured 5, 15, $30 \mathrm{~min}, 1,2 \mathrm{and} 4 \mathrm{~h}$ after plating. $\mathrm{N}=5$. e Confocal images of ypet-tagged talin WT or ypet-tagged talin DM cells on FN-coated round micropatterns stained for paxillin (red) and F-actin (blue). Ypet signal is shown in green. Scale bar $10 \mu \mathrm{m}$. Focal adhesion area per cell area (f), focal adhesion number per cell (g), and focal adhesion size (h) of ypettagged talin WT and ypet-tagged talin DM cells on FN-coated micropatterns. i Ypet fluorescence intensity within paxillin-positive focal adhesion area in relation to the cellular ypet fluorescence. $N=5 ; 10-15$ talin WT and talin DM-expressing cells in each measurement were analyzed. All values are given as mean \pm S.E.M. ${ }^{*} p<0.05$ and ${ }^{*} p<0.01$
} 
a b
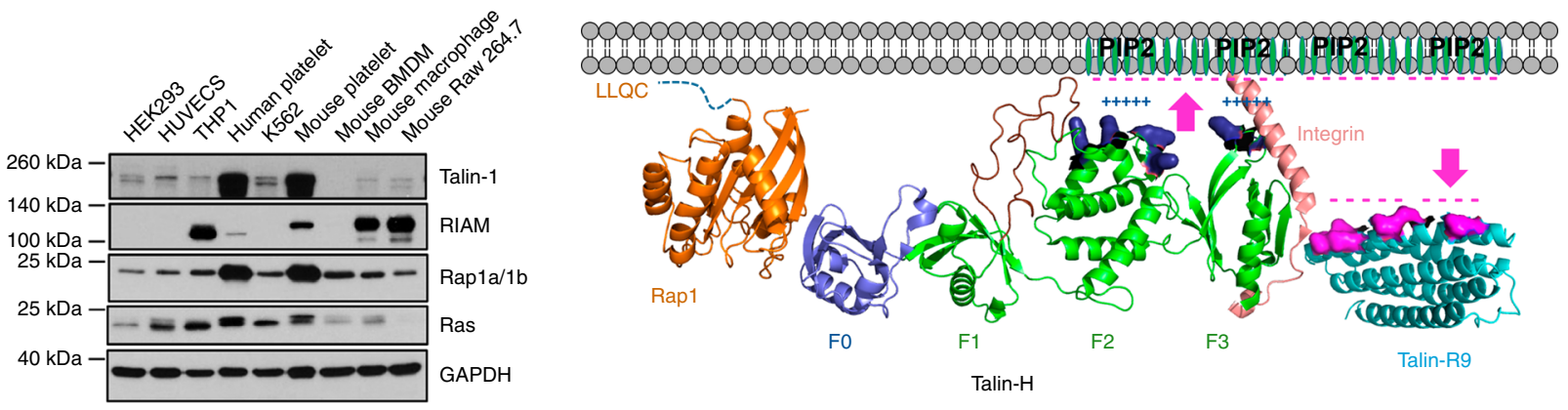

C

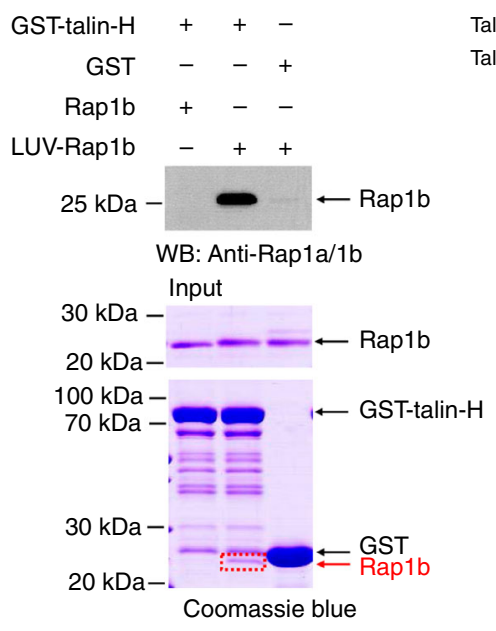

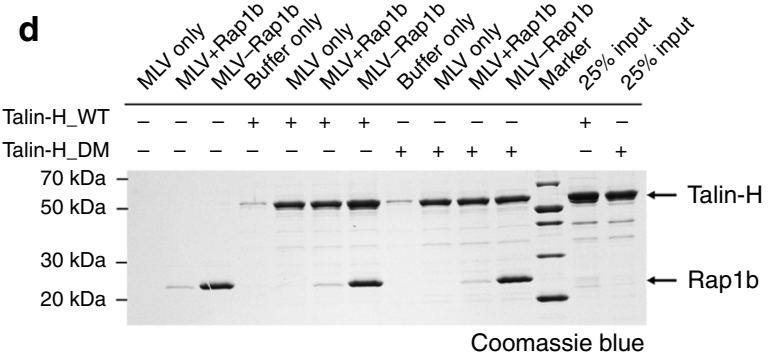

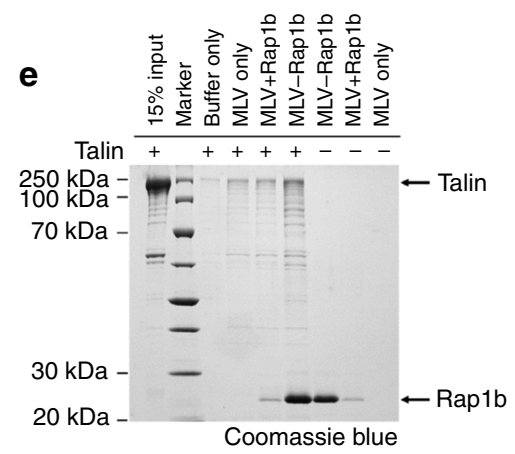

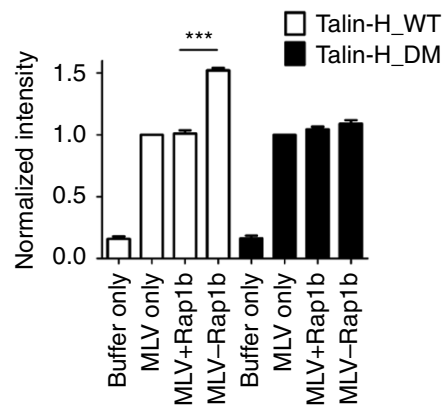

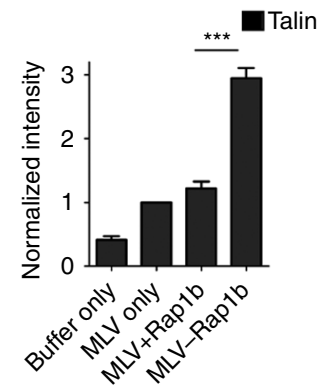

Fig. 6 Mechanism of talin recruitment by Rap1. a Protein expression levels of talin, RIAM, Rap1, Ras GTPases, and GAPDH in various types of cell lines indicated in the figure. Full blots are shown in Supplementary Fig. 13. b A model of membrane-associated Rap1b recruiting talin for integrin activation. Proteins are shown in cartoon representation. Talin F1 loop is colored in maroon, the positively charged residues of talin-F2F3 domains (green), which interact with PIP2, are colored in deep blue and shown in surface representation, and the negatively charged residues of talin-R9 (cyan) are colored in magenta and shown in surface representation. c GST pull-down assay to show the robustly enhanced interaction between membrane-anchored Rap1b and talin-H. Rap1b was loaded with GMP-PNP in this assay and "LUV-Rap1b" represents that Rap1b was anchored to large unilamellar vesicles (LUVs). LUVRap1b could be easily pulled down by GST-talin-H and the band was visible in Coomassie blue staining gel (boxed in red) and intense in WB analysis, while free Rap1b pulled down by GST-talin-H was hardly seen in the same condition. Full blot/gel images are shown in Supplementary Fig. 14. d Left, a representative vesicle co-sedimentation assay showing that the interaction between talin-H_WT and membrane is enhanced by around 1.5-folds when Rap1b (GMP-PNP) is attached to membrane but not for talin-H_DM. "MLV + Rap1b" represents that Rap1b remained as free form in solution without being attached to multilamellar vesicles (MLVs), which were pre-incubated with $\beta$-mercaptoethanol. "MLV-Rap1b" represents that Rap1b was anchored to MLVs. Right, the quantification of four independent experiments. The intensity of talin-H_WT or talin-H_DM band was normalized to that of corresponding "MLV only" group and the data are shown in means \pm S.E.M. ${ }^{\star \star \star} p<0.0001$. Full gel image is shown in Supplementary Fig. 15. e Left, a representative vesicle cosedimentation assay showing that the interaction between full-length talin and membrane is significantly enhanced by around 3-folds when Rap1b (GMPPNP) is attached to membrane. Right, the quantification of four independent experiments. The intensity of talin band was normalized to that of the "MLV only" group and the data are shown as means \pm S.E.M. ${ }^{\star \star \star} p<0.0001$. Full gel image is shown in Supplementary Fig. 15

biological processes ${ }^{45}$. Interestingly, recent proteomic analyses have suggested that weak PPIs dominate in these cellular PPI networks ${ }^{46}$. However, because cellular protein concentrations, which are typically estimated on whole-cell volumes, are low (e.g., $\mathrm{nM}$ ), weak binary PPIs, as detected by conventional in vitro methods, were commonly treated to be nonspecific and therefore disregarded for further investigations. Nevertheless, these weak PPIs may be enhanced and they exert crucial functions at specific microenvironments, which is strongly demonstrated in our study. Specifically, while isolated Rap1b and talin-F0 binds at sub-mM affinity, the binding became very strong in the presence of membrane that mimics the cellular microenvironment. As illustrated in Fig. 6b, the membrane-anchoring ability of both Rap1b and talin may increase the local concentration of both proteins, thereby markedly strengthening their association. Other types of cellular strategies may also exist to enhance the association of otherwise weak binary interactions, which remain to be further investigated. With the completion of human genome and rapidly growing data of weak PPIs ${ }^{46}$, our study emphasizes cautious treatment of a wide variety of weak PPIs by including specific microenvironments. In this regard, NMR is particularly valuable for deciphering the detailed structural basis of weak $\mathrm{PPIs}^{32}$ in vitro, laying down the foundation for further analysis of the interaction in certain networks both in vitro and in vivo, as 
exemplified in our study. Such approach would complement with other techniques such as crystallography and CryoEM that are advantageous for elucidating large high-affinity complexes, together, facilitating a thorough and unbiased view of how proteins function in cells.

\section{Methods \\ Plasmid constructs and mutagenesis. The following constructs for bacterial} expression were used in this study: mouse talin-F0 (1-86) subcloned into a pHis-1 vector, mouse full-length talin subcloned into a pET28t vector, human kindlin2-F0 (1-105) subcloned into a pGST-1 vector, mouse talin-H (1-429) subcloned into a pET28t, and a pGST-1 vector, mouse RIAM-RAPH (149-438) subcloned into a pGST-1 vector, human full-length Raplb subcloned into a pGST-1 vector, and Cterminal truncated Rap1b (1-167) subcloned into a pET28t vector. Human wildtype Rap1b and H-Ras (G12V) subcloned into pET28a vectors (6xHis tag) were kind gifts from Dr. Matthias Buck at Case Western Reserve University. Construct mutagenesis was conducted by using QuickChange lightning site-directed mutagenesis Kit (Agilent Technologies). Mutated constructs generated in this study include talin-F0_DM (K15A, R35A), talin-H_DM (K15A, R35A), Rap1b (G12V), Raplb (1-167, G12V), Rap1b (G12V, I27H), Rap1b (G12V, K31E), Rap1b_TM1 (C51S, C118S, C141S), and Rap1b_TM2 (G12V, C51S, C118S, C141S). Note that pET28t is a modified pET28a vector where the thrombin cleavage site is substituted with a TEV protease cleavage site. Primers used in this study are listed in Supplementary Table 3 .

Protein expression and purification. Recombinant proteins with fusion tag (6xHis tag or GST tag) were expressed in Escherichia coli BL21 (DE3) strain (New England Biolabs). Typically, bacteria was initially grown in $50 \mathrm{ml}$ Lysogeny broth (LB) medium and then amplified in $1 \sim 21 \mathrm{LB}$ at $37^{\circ} \mathrm{C}$. The culture was induced by $0.4 \mathrm{mM}$ Isopropyl $\beta$-D-1-thiogalactopyranoside (IPTG) at $20^{\circ} \mathrm{C}$ or room temperature (RT) for overnight when it reached an $\mathrm{A}_{600}$ of 0.7 . The pellet was then collected and suspended in buffer and frozen at $-80^{\circ} \mathrm{C}$. For protein purification, the pellet was lysed by incubation with lysozyme and sonication. After high-speed centrifugation, the supernatant was subjected to affinity column purification by using either nickel or GST gravity column. Ion exchange chromatography was an optional purification step depending on protein purity (in this study, full-length Raplb was purified by anion exchange method using Hi-trap Q column). Gel filtration was always performed in the final step by using either Superdex-75 (16/ $60)$ or Superdex-200 (16/60; GE Healthcare). Note that Superose-6 (10/300) was used for full-length talin purification. Purified protein was checked by SDS-PAGE. Proteins with $90 \%$ purity or above were used in this study. According to experimental requirement, fusion tag (either His-tag or GST tag) was removed by TEV protease during protein purification if necessary. Isotope-labeled $\left({ }^{15} \mathrm{~N}\right.$ and/or $\left.{ }^{13} \mathrm{C}\right)$ proteins were achieved by employing minimal medium with ${ }^{15} \mathrm{NH}_{4} \mathrm{Cl}$ and/or ${ }^{13} \mathrm{C}$ glucose as the sole nitrogen and carbon sources. ${ }^{2} \mathrm{H}$-labeled protein was achieved by using the minimal medium prepared in $99.8 \% \mathrm{D}_{2} \mathrm{O}$ with ${ }^{2} \mathrm{H}$-glucose as the carbon source. Protein concentration was measured by absorbance at $280 \mathrm{~nm}$ or Pierce $660 \mathrm{~nm}$ protein assay reagent (Thermo Fisher Scientific).

GTPase nucleotide exchange. Non-hydrolyzable analogs of GDP and GTP, named Guanosine $5^{\prime}$ - $[\beta$-thio] diphosphate trilithium salt (GDP- $\beta$-S) and Guanosine $5^{\prime}$ - $[\beta, \gamma$-imido] triphosphate trisodium salt (GMP-PNP), respectively, were purchased from Sigma-Aldrich. In this study, wild-type Rap1b loaded with GDP- $\beta$ $\mathrm{S}$ was used as an inactive form, and Raplb (G12V) or H-Ras (G12V) loaded with GMP-PNP was used as an active form. Purified wild-type Raplb $(50 \mu \mathrm{M})$, Raplb $(\mathrm{G} 12 \mathrm{~V})$ or H-Ras (G12V) was first incubated with 2 mM EDTA and 2 mM GDP- $\beta$ $\mathrm{S}$ or GMP-PNP at RT for $35 \mathrm{~min}, \mathrm{MgCl}_{2}$ was then added in to reach a final concentration of $7 \mathrm{mM}$ and incubated for another $30 \mathrm{~min}$. After that, they were kept on ice and concentrated into a high concentration for experimental use.

NMR 2D-HSQC and HSQC titration. 2D-HSQC experiments were performed on Bruker $600 \mathrm{MHz}$ NMR spectrometer, and samples containing $50 \mu \mathrm{M}{ }^{15} \mathrm{~N}$-labeled protein were studied. For ${ }^{15} \mathrm{~N}$-labeled talin-F0 or kindlin2-F0, experiments were performed at $25^{\circ} \mathrm{C}$ in buffer containing $20 \mathrm{mM} \mathrm{NaH} \mathrm{PO}_{4} / \mathrm{Na}_{2} \mathrm{HPO}_{4}(\mathrm{pH}$ 6.6), 50 $\mathrm{mM} \mathrm{NaCl}, 5 \mathrm{mM} \mathrm{MgCl}_{2}, 2 \mathrm{mM}$ dithiothreitol (DTT), and 5\% $\mathrm{D}_{2} \mathrm{O}$. For ${ }^{15} \mathrm{~N}$-labeled Raplb (1-167), experiments were performed at $25^{\circ} \mathrm{C}$ in buffer containing $25 \mathrm{mM}$ $\mathrm{NaH}_{2} \mathrm{PO}_{4} / \mathrm{Na}_{2} \mathrm{HPO}_{4}$ (pH 6.8), $150 \mathrm{mM} \mathrm{NaCl}, 5 \mathrm{mM} \mathrm{MgCl}, 2 \mathrm{mM}$ DTT, and 5\% $\mathrm{D}_{2} \mathrm{O}$. Chemical shift change $\left(\Delta \delta_{\text {obs }[\mathrm{HN}, \mathrm{N}]}\right)$ was calculated with the equation $\Delta \delta_{\text {obs }}$ $\left.[\mathrm{HN}, \mathrm{N}]=\left[\left(\Delta \delta_{\mathrm{HN}} W_{\mathrm{HN}}\right)^{2}+\left(\Delta \delta_{\mathrm{N}} W_{\mathrm{N}}\right)^{2}\right)\right]^{1 / 2}$, where $W_{\mathrm{HN}}$ and $W_{\mathrm{N}}$ are weighting factors based on the gyromagnetic ratios of ${ }^{1} \mathrm{H}$ and ${ }^{15} \mathrm{~N}\left(W_{\mathrm{HN}}=1\right.$ and $\left.W_{\mathrm{N}}=0.154\right)$ and $\Delta \delta$ (p.p.m.) $=\delta_{\text {bound }}-\delta_{\text {free. }}$ For HSQC titration, samples containing $45 \mu \mathrm{M}$ ${ }^{15} \mathrm{~N}$-labeled talin-F0 with increasing amount of GMP-PNP-loaded Rap1b (G12V) were used to collect 2D-HSQC spectra. Dissociation constant $\left(K_{\mathrm{d}}\right)$ was achieved by fitting the chemical shift changes into the equation as shown below ${ }^{47}$. Four independent well-resolved residues were selected for fitting and an average $K_{\mathrm{d}}$ was obtained.
$\Delta \delta_{\text {obs }}=\Delta \delta_{\max }\left\{\left(K_{\mathrm{d}}+[\mathrm{P}]_{\mathrm{t}}+[\mathrm{L}]_{\mathrm{t}}\right)-\left[\left(K_{\mathrm{d}}+[\mathrm{P}]_{\mathrm{t}}+[\mathrm{L}]_{\mathrm{t}}\right)^{2}-4 \times[\mathrm{P}]_{\mathrm{t}} \times[\mathrm{L}]_{\mathrm{t}}\right]^{1 / 2}\right\} / 2[\mathrm{P}]_{\mathrm{t}}$ $\left(\Delta \delta_{\max }\right.$, maximum chemical shift change; $[\mathrm{P}]_{\mathrm{t}}$, labeled protein concentration; $[\mathrm{L}]_{\mathrm{t}}$, ligand or titrant concentration).

GST pull-down. A volume of $\sim 25 \mu \mathrm{g}$ purified GST or GST-fused protein was immobilized on $15 \mu$ l glutathione-Sepharose $4 \mathrm{~B}$ resin (beads) via incubation on a rotator for $1.5 \mathrm{~h}$ at $4{ }^{\circ} \mathrm{C}$ in the binding buffer containing $25 \mathrm{mM}$ Tris- $\mathrm{HCl}(\mathrm{pH} \mathrm{7.5)}$, $150 \mathrm{mM} \mathrm{NaCl}, 5 \mathrm{mM} \mathrm{MgCl}$, $1 \mathrm{mM}$ DTT, $0.01 \%$ Nondiet P-40, and supplemented with Complete EDTA-free Protease Inhibitor (Roche, Indianapolis, IN). Desired amount of prey protein was then added in and incubated with beads for another 2 $\mathrm{h}$ at $4{ }^{\circ} \mathrm{C}$. After that, the beads were washed by $600 \mu$ l binding buffer twice and subjected to be denatured by adding $35 \mu \mathrm{l} 2 \times \mathrm{SDS}$ loading buffer and boiling for 5 min. After centrifuging down, supernatants were resolved by SDS-PAGE or western blotting. All the pull-down experiments were performed in triplicate independently.

Western blotting and antibodies. After SDS-PAGE, samples were transferred onto a $0.45 \mu \mathrm{m}$ polyvinylidene fluoride (PVDF) membrane (Millipore, Billerica, MA). The membrane was blocked with $5 \%$ non-fat milk in TBST buffer overnight at $4{ }^{\circ} \mathrm{C}$. After that, the membrane was incubated with primary antibody at RT for 3 $h$ and then incubated with horeseradish peroxidase (HRP)-conjugated secondary antibody at RT for $1 \mathrm{~h}$. The blots were detected by Pierce ECL Western Blotting Substrate (Thermo Fisher Scientific). The following primary antibodies were used in this study: 6xHis epitope tag antibody (Catalog\# MA1-21315, Thermo Fisher Scientific), Rapla/Raplb (26B4) Rabbit mAb (Catalog\# 2399, Cell Signaling Technology), Ras (27H5) Rabbit mAb (Catalog\# 3339, Cell Signaling Technology), GAPDH (D16H11) Rabbit mAb (Catalog\# 5174, Cell Signaling Technology), AntiTalin-1 antibody (Catalog\# ab57758, Abcam Inc.), and Anti-RIAM antibody (EPR2806; Catalog\# ab92537, Abcam Inc.). The following secondary antibodies were used in this study: anti-mouse IgG, HRP-linked antibody (Catalog\# 7076, Cell Signaling Technology), and anti-rabbit IgG, HRP-linked antibody (Catalog\# 7074, Cell Signaling Technology). Primary antibodies were used at 1:1000 dilution, and secondary antibodies were used at 1:3000 dilution.

Assignment of Rap1b and talin-FO and their bound forms. C-terminal truncated Rap1b (1-167) with G12V mutation was used in all the following structure determination studies. For assignment of Rap $1 \mathrm{~b},{ }^{15} \mathrm{~N} /{ }^{13} \mathrm{C}$-labeled Rap1b was purified and the N-terminal $6 \mathrm{xHis}$ tag was removed during purification. Standard triple-resonance experiments were conducted with 0.6 mM GMP-PNP-loaded Rap1b. Rap1b sequential assignment was performed using PASA software ${ }^{48}$. For assignment of talin-F0, the complete chemical shift-assignment table of talin-F0 from Biological Magnetic Resonance Data Bank (accession code 15458) was downloaded and used in this study ${ }^{26}$. Amide proton to nitrogen to $\alpha$-carbon correlation (HNCA) experiment was performed on $1.0 \mathrm{mM}{ }^{15} \mathrm{~N} /{ }^{13} \mathrm{C}$-labeled talinF0 to confirm the assigned resonances. All these experiments were performed on Bruker $600 \mathrm{MHz}$ NMR spectrometer, and at $25^{\circ} \mathrm{C}$ in the buffer that contained 20 $\mathrm{mM} \mathrm{NaH}{ }_{2} \mathrm{PO}_{4} / \mathrm{Na}_{2} \mathrm{HPO}_{4}$ (pH 6.6), $50 \mathrm{mM} \mathrm{NaCl}, 5 \mathrm{mM} \mathrm{MgCl} 2,2 \mathrm{mM}$ d-DTT, and $5 \% \mathrm{D}_{2} \mathrm{O}$. For assignments of the bound Raplb and talin-F0 forms, the chemical shifts of majority of residues were readily transferred from the free forms with slight adjustments as they are similar to those of the free forms. A few residues (e.g., I27, R41) exhibited duplex peaks in the free form of Rap1 likely due to local conformational flexibility, and most of these peaks were merged into single peaks in the complex. All assignments were also verified by through-bond NOESY experiments of the complex.

3D-NOESY experiments. The following samples were prepared: (1) $0.6 \mathrm{mM}{ }^{15} \mathrm{~N} /$ ${ }^{13} \mathrm{C}$-labeled Raplb in the presence of $0.9 \mathrm{mM}$ unlabeled talin-F0, (2) $0.5 \mathrm{mM}{ }^{15} \mathrm{~N} /$ ${ }^{13} \mathrm{C}$-labeled talin-F0 in the presence of $0.7 \mathrm{mM}$ unlabeled Raplb, (3) $0.5 \mathrm{mM}{ }^{15} \mathrm{~N} /$ $100 \%{ }^{2} \mathrm{H}$-labeled talin-F0 in the presence of $0.7 \mathrm{mM}$ unlabeled Rap1b, and (4) 0.5 $\mathrm{mM}{ }^{15} \mathrm{~N} /{ }^{13} \mathrm{C}$-labeled talin-F0 in the presence of $0.7 \mathrm{mM}$ unlabeled Rap1b prepared in $99.8 \% \mathrm{D}_{2} \mathrm{O}$. The following NOESY experiments were performed using the above samples: (i) $3 \mathrm{D}{ }^{15} \mathrm{~N} /{ }^{13} \mathrm{C}$-edited NOESY experiment (120 ms mixing time) with sample 1, (ii) $3 \mathrm{D}{ }^{15} \mathrm{~N} /{ }^{13} \mathrm{C}$-edited NOESY experiment (120 ms mixing time) with sample 2, (iii) $3 \mathrm{D}^{15} \mathrm{~N}$-edited NOESY experiment (300 ms mixing time) with sample 3 , and (iv) $3 \mathrm{D}{ }^{15} \mathrm{~N} /{ }^{13} \mathrm{C}$-filtered NOESY experiment (120 ms mixing time) with sample 4. All these experiments were performed on Bruker 850 or $900 \mathrm{MHz}$ spectrometers at the same condition as mentioned above.

Structure determination. All NMR data were processed and analyzed using nmrPipe ${ }^{49}$, PIPP $^{50}$, and Sparky ${ }^{51}$. Distance restraints (NOE constraints) were obtained from 3D-NOESY experiments mentioned above, and Xplor-NIH ${ }^{52}$ was used to calculate the complex structure. In brief, the individual structures of Rap1b and talin-F0 in complex form were calculated first based on the NOEs collected from sample 1 (2308 NOEs) and sample 2 (1114 NOEs), respectively. 302 dihedralangle restraints for Rap $1 \mathrm{~b}$ derived from $\mathrm{TALOS}^{53}$ were applied during Rap $1 \mathrm{~b}$ structure calculation. The initial templates for Raplb and talin-F0 were derived from PDB database with PDB codes of 4DXA and 3IVF, respectively. A total of 78 unambiguous intermolecular NOEs collected from sample 3 and sample 4 were used to calculate complex structure (also see Supplementary Table 1 for structural 
statistics). After refinements, a total of 50 final structures were calculated and the 20 lowest-energy structures were selected for analysis. The quality of structure was evaluated with PROCHECK ${ }^{54}$. Structures are visualized by PyMOL 1.3 (Schrödinger LLC.).

Membrane-anchored Rap1b preparation. To prepare large unilamellar vesicles (LUVs), 1-palmitoyl-2-oleoyl-sn-glycero-3-phosphocholine (POPC), L- $\alpha$-phosphatidylinositol-4,5-bisphosphate $\left(\mathrm{PI}(4,5) \mathrm{P}_{2}\right)$, and 1,2-dipalmitoyl-sn-glycero-3phosphoethanolamine- $\mathrm{N}$-[4-(p-maleimidomethyl)cyclohexane-carboxamide] (16:0 PE MCC) were purchased from Avanti Polar Lipids. LUVs, which consist of $96 \%$ POPC, $1 \% \mathrm{PIP}_{2}$, and $3 \%$ PE MCC, were prepared by extrusion. In brief, lipids were first dissolved together in chloroform, and then the chloroform was removed under a stream of nitrogen followed by overnight vacuum pumping. The lipid film was suspended in buffer containing $25 \mathrm{mM} \mathrm{NaH} \mathrm{PO}_{4} / \mathrm{Na}_{2} \mathrm{HPO}_{4}$ (pH 6.8), $100 \mathrm{mM}$ $\mathrm{NaCl}$, and $5 \mathrm{mM} \mathrm{MgCl}$, and subjected to homogenization with a few freeze-thaw cycles. LUVs were finally formed by extruding the lipid suspension $\sim 20$ times through two stacked $0.1 \mathrm{~mm}$ polycarbonate filters. Rap $1 \mathrm{~b}$ was tethered to LUVs via Michael addition between the thiol group of Cys 180 and the maleimide group of PE MCC ${ }^{41,55}$. To mimic the physiological condition that only the C-terminal Cys 180 of Raplb is attached to membrane and meanwhile to avoid nonspecific chemical interactions, all the cysteines of Rap1b except Cys 180 were mutated to serines. Rap1b_TM1 (C51S, C118S, and C141S) and Rap1b_TM2 (G12V, C51S, C118S, and C141S) were generated, purified, and loaded with GDP- $\beta$-S and GMPPNP, respectively, as described above. Rap1b_TM2 was first confirmed to interact with ${ }^{15} \mathrm{~N}$-talin-F0 by HSQC (Supplementary Fig. 10a). To make Rap1b-anchored LUVs, Rap1b_TM1 or Rap1b_TM2 was incubated with freshly prepared LUVs in 1:6 molar ratio overnight at RT in the same buffer. The reaction was terminated by adding $\beta$-mercaptoethanol to a final concentration of $5 \mathrm{mM}$. The achievement of membrane-anchored Rap1b was confirmed by Native-PAGE combined with SDSPAGE (Supplementary Figs. 10b and 20). Unanchored Rap1b was dialyzed out in the same buffer using a dialyzer with $100 \mathrm{kDa}$ cutoff membrane (Harvard Apparatus) before use.

Vesicle co-sedimentation. Multilamellar vesicles (MLVs) rather than LUVs are used in this study since MLVs are larger in size and could be pelleted down easily. MLVs, which consist of 87\% POPC, 10\% PIP2, and 3\% PE MCC were prepared similarly to LUVs as described above but without extrusion. MLVs with anchored Raplb (either GDP- $\beta$-S or GMP-PNP) were also prepared similarly as described above. Talin- $\mathrm{H}(10 \mu \mathrm{M})$ or full-length talin was incubated with $10 \mu \mathrm{M}$ "MLVRap1b (Rap1b-anchored MLVs)", "MLV only", and "MLV + Rap1b (MLVs with unanchored Raplb)" separately in a " $7 \times 21 \mathrm{~mm}$ " Polycarbonate tube (Beckman Coulter Inc.) with a total volume of $30 \mu \mathrm{l}$ at RT for $15 \mathrm{~min}$. Samples were then centrifuged down at 22,000 r.p.m. for $35 \mathrm{~min}$. Membrane pellet was dissolved in $2 \times$ SDS loading buffer and subjected to SDS-PAGE analysis. Gels were stained with Coomassie blue, and then scanned and quantified with an Odyssey CLx Imaging System (LI-COR Inc.). The intensities of protein bands were quantified by the fluorescent signal detected from the $700 \mathrm{~nm}$ channel according to the manufacturer's protocol. Experiments were performed in quadruplets. Statistical significance was tested by two-tailed unpaired $t$-test.

Isothermal titration calorimetry. ITC experiments were performed using a Microcal iTC 200 system (GE Healthcare Life Sciences) at $25^{\circ} \mathrm{C}$. Before experiments, all samples were dialyzed into the same buffer that contained $25 \mathrm{mM}$ $\mathrm{NaH}_{2} \mathrm{PO}_{4} / \mathrm{Na}_{2} \mathrm{HPO}_{4}(\mathrm{pH} 6.8), 100 \mathrm{mM} \mathrm{NaCl}$, and $5 \mathrm{mM} \mathrm{MgCl}$ overnight at $4{ }^{\circ} \mathrm{C}$. Talin-H $(200 \mu \mathrm{M})$ in the syringe $(\sim 40 \mu \mathrm{l})$ was injected 20 times in $2.0 \mu \mathrm{l}$ aliquots into the sample-cell $(\sim 220 \mu \mathrm{l})$ containing $15 \mu \mathrm{M}$ LUV-Raplb (GMP-PNP). As controls (Supplementary Fig. 10c), talin-H was titrated into LUV only, Rap1b only, or buffer only, and talin-H_DM was titrated into LUV-Rap1b (GMP-PNP) using the same condition. ITC Data were analyzed by fitting to a single-site binding model with Origin Software.

Microscale thermophoresis analysis. The affinity of the interaction between GMP-PNP-loaded GFP-Rap1b (G12V) and RIAM-RAPH, talin-F0 or talin-H was measured by using a Monolith NT.115 instrument (NanoTemper Technologies). Rap1b (G12V) was subcloned into a pRSET vector (Thermo Fisher Scientific), which expresses an N-terminal 6xHis tag and a C-terminal GFP fusion tag. RIAM$\mathrm{RAPH}$, talin-F0, or talin-H was prepared at different concentrations through 1:1 serial dilution and then mixed with $100 \mathrm{nM}$ GFP-Rap1b (G12V) in buffer containing $50 \mathrm{mM}$ Tris- $\mathrm{HCl}$ ( $\mathrm{pH} 7.5$ ), $100 \mathrm{mM} \mathrm{NaCl}, 5 \mathrm{mM} \mathrm{MgCl}, 2 \mathrm{mM}$ DTT, and $0.05 \%$ Tween 20 . The samples were incubated at RT for $10 \mathrm{~min}$ and then loaded into microscale thermophoresis (MST) standard or premium capillary tubes. The fluorescent signal of GFP fusion tag was detected by the blue channel of the instrument according to the manufacturer's protocol, and the data were collected using 20\% LED power and 40\% MST powder at RT. Three independent experiments were performed, and the collected MST data were used to fit the dissociation constant $\left(K_{\mathrm{d}}\right)$ by using the MO. Affinity Analysis software (NanoTemper Technologies).
Integrin activation assay. Wild-type talin-H or talin-H_DM (K15A, R35A) was subcloned into a pEGFP-C1 vector. CHO A5 cells stably expressing integrin $\alpha_{\mathrm{IIb}} \beta_{3}$ were transfected with empty vector, wild-type talin-H, or talin-H_DM using Lipofectamine 2000 reagent (Life Technologies). After $24 \mathrm{~h}$, cells were stained with anti- $\alpha_{\text {Ib }} \beta_{3}$ activation-specific mAb PAC1 (1:100 dilution; Catalog\# 340535, BD Biosciences) at RT for $40 \mathrm{~min}$, and later incubated with Alexa Flour 647 Goat AntiMouse IgM (Catalog\# 115-607-020, Jackson Immunology Laboratories; 1:3000 dilution) on ice for $30 \mathrm{~min}$. After wash, cells were fixed and subjected to flow cytometry analysis. Values for PAC1 binding were reported as median fluorescent intensities and the data were normalized to control with empty vector only and are presented by the means \pm S.E.M. The two-tailed unpaired $t$-test was performed to calculate the $p$ value using GraphPad software.

Generation of cell lines and cell culture conditions. Murine talin 1 and 2 doubleknockout fibroblasts $\left(\operatorname{talin}^{1 / 2 \mathrm{dko}}\right)^{38}$ were retrovirally transduced with pLPCX expression construct containing YPET alone, C terminally YPET-tagged talin-1 cDNA (talin WT), or YPET-tagged double-mutant talin-1 (talin DM; K15A, R35A) using the Phoenix retroviral expression system. Phoenix cells were transfected using a conventional calcium phosphate protocol and five infection cycles were performed by transferring the phoenix cell culture supernatant supplemented with $5 \mathrm{\mu g} \mathrm{ml}^{-1}$ polybrene to talin ${ }^{1 / 2 \mathrm{dko}}$ cells ${ }^{56,57}$. Cells were FACS-sorted using a FACSAria $^{\text {tw }}$ IIu cell sorter (BD Biosciences, Heidelberg, Germany) to isolate cells with comparable expression levels. Cells were characterized by western blotting using mouse $\alpha$-GFP (home-made), rabbit anti-talin-1(Catalog\# sc-15336, Santa Cruz Biotechnology Inc.), rabbit anti-Rap1 (Catalog\# sc-65, Santa Cruz Biotechnology Inc.), rabbit anti-RIAM (Catalog\# ab92537, Abcam Inc.), mouse antiGAPDH (Catalog\# CB1001-500UG, Millipore), goat anti-rabbit-HRP (Catalog\# 111-035-144, Jackson ImmunoResearch Laboratories), and goat anti-mouse-HRP (Catalog\# 115-035-003, Jackson ImmunoResearch Laboratories Inc.) antibodies following standard protocols. Rabbit anti-talin-1, rabbit anti-Rap1, and rabbit antiRIAM antibodies were used at a dilution of 1:1000. Mouse anti-GAPDH, goat antirabbit-HRP, and goat anti-mouse-HRP antibodies were used at a dilution of 1:20,000. Cells were cultured under standard conditions in DMEM GlutaMAX ${ }^{\mathrm{TM}}$ (Thermo Fisher Scientific) supplemented with 10\% fetal bovine serum, $100 \mathrm{U} \mathrm{ml}^{-1}$ penicillin, $100 \mathrm{\mu g} \mathrm{ml}^{-1}$ streptomycin, and non-essential amino acids (all from Thermo Fisher Scientific).

Adhesion and spreading assays. For adhesion assays, polystyrol flat-bottom 96well microplates (Greiner Bio-One, Frickenhausen, Germany) were coated with 10 $\mu \mathrm{g} \mathrm{ml}^{-1}$ laminin (Sigma) in HBSS (Thermo Fisher Scientific), $5 \mu \mathrm{g} \mathrm{ml}^{-1} \mathrm{FN}, 0.01 \%$ poly-L-lysine (both Sigma), or $5 \mu \mathrm{g} \mathrm{m}^{-1}$ VN (STEMCELL Technologies, Köln, Germany) in coating buffer (20 mM Tris-HCl pH 9.0, $150 \mathrm{mM} \mathrm{NaCl}, 2 \mathrm{mM}$ $\mathrm{MgCl}_{2}$ ) overnight at $4{ }^{\circ} \mathrm{C}$. After blocking with $3 \%$ bovine serum albumin in PBS for $30 \mathrm{~min}$ at room temperature, $4 \times 10^{4}$ cells in DMEM containing $0.1 \% \mathrm{FBS}$, and 25 $\mathrm{mM}$ HEPES were seeded per well, incubated for $1 \mathrm{~h}$, and washed with PBS. Adherent cells were fixed with $4 \%$ paraformaldehyde (PFA) for $15 \mathrm{~min}$ and stained with $5 \mathrm{mg} \mathrm{ml}^{-1}$ crystal violet in $2 \%$ ethanol for $30 \mathrm{~min}$. After washing, remaining crystal violet was dissolved in $2 \%$ SDS and quantified by measuring absorbance at $595 \mathrm{~nm}$ using a microplate reader (Tecan, Männedorf, Switzerland). All experiments were performed in quadruplets. For spreading analysis, cells were plated on FN $\left(5 \mu \mathrm{g} \mathrm{ml}^{-1}\right)$-coated dishes and phase contrast pictures were taken using an EVOS $^{\mathrm{TM}}$ FL Auto life cell microscope (Thermo Fisher Scientific) 5, 15, 30, 60, 120, and $240 \mathrm{~min}$ after seeding the cells. Cell spreading area of 30 cells per group at each time point were measured using ImageJ software (US National Institutes of Health). Statistical significance was tested by two-tailed paired $t$-test.

Flow cytometry. Integrin surface expression was determined by FACS using a LSRFortessa $^{\mathrm{TM}} \mathrm{X}$-20 flow cytometer (BD Biosciences). Staining and measurement were performed in PBS supplemented with $2 \%$ FBS and 2 mM EDTA. Cells were incubated with biotinylated anti-integrin antibodies and subsequently with Cy5labeled strepdavidin (Catalog\# 016-170-084, Jackson ImmunoResearch Laboratories). Data were analyzed using FlowJo software. The following antibodies were used for flow cytometry: hamster IgM anti-integrin $\beta 1$ (Catalog\# 13-0291-82, eBioscience), rat IgG2a anti-integrin $\alpha 6$ (Catalog\# 13-0495-82, eBioscience), rat IgG1 isotype control (Thermo Fisher Scientific), hamster IgG isotype control (Thermo Fisher Scientific), hamster IgG anti-integrin $\beta 3$ (Catalog\# 553345, BD Pharmingen), rat IgG2a anti-integrin $\alpha 5$ (Catalog\# 557446, BD Pharmingen ${ }^{\mathrm{TM}}$ ), rat IgGl anti-integrin $\alpha \mathrm{V}$ (Catalog \#104104, BioLegend), hamster IgM isotype control (BioLegend), and rat IgG2a isotype control (BioLegend). All listed antibodies were biotinylated and used at 1:200 dilution.

Immunostaining and FA analysis. Cells were cultured on FN-coated glass coverslips (Thermo Fisher Scientific) overnight or on FN-coated micropatterns (discshaped with $1100 \mu^{2}$ area; Cytoo, Grenoble, France) for $5 \mathrm{~h}$ and fixed for $10 \mathrm{~min}$ with 4\% PFA. Mouse anti-paxillin with a dilution of 1:300 (Catalog\# 610051, BD Transduction Laboratories, Heidelberg, Germany), goat anti-mouse-Cy3 with a dilution of 1:400 (Catalog\# 115-165-146, Jackson ImmunoResearch Laboratories), and Phalloidin-Alexa Fluor 647 with a dilution of 1:100 (Catalog\# A22287, Thermo Fisher Scientific) were used for immunostainings. Images were acquired using a 
Leica TCS SP5 X confocal microscope (Leica Microsystems, Wetzlar, Germany) equipped with $\times 63$ numerical aperture (NA) 1.40 oil objective lenses and Leica Confocal Software (LAS AF). All pictures were processed with Photoshop (Adobe Systems, San José, CA, USA). FA area was defined by paxillin staining and quantified using ImageJ software. For quantitative analysis of talin recruitment to FAs, the total ypet signal intensity within the FA area was normalized to the total ypet fluorescence of the whole cell. Statistical significance was tested by two-tailed paired $t$-test.

Cell lysate preparations. Various types of cells indicated in Fig. 6a were isolated from blood or cultured cells. Cells were washed once with cold PBS, and lysed by adding buffer that contained $50 \mathrm{mM}$ Tris- $\mathrm{HCl}(\mathrm{pH}$ 6.8) and $1 \%$ SDS. The cell lysates were boiled for $5 \mathrm{~min}$, and the concentration of total protein in each cell lysate was quantified with Pierce BCA Protein Assay Kit (Thermo Fisher Scientific). Approximately $8 \mu \mathrm{g}$ total protein for each lysate was loaded onto SDS-PAGE gels for western blotting analysis.

Data availability. Accession codes: The complete chemical shift-assignment tables of free Rap1b, bound Rap1b, and bound talin-F0 have been deposited in the Biological Magnetic Resonance Bank (BMRB) with the accession code 30353. The coordinates of Rap1b/talin-F0 complex structure have been deposited in the Protein Data Bank (PDB) with the code 6BA6. Other data are available from the corresponding authors upon reasonable request.

Received: 11 April 2017 Accepted: 18 October 2017

Published online: 23 November 2017

\section{References}

1. Springer, T. A. Adhesion receptors of the immune system. Nature 346, 425-434 (1990)

2. Hynes, R. O. Integrins: bidirectional, allosteric signaling machines. Cell 110, 673-687 (2002)

3. Qin, J., Vinogradova, O. \& Plow, E. F. Integrin bidirectional signaling: a molecular view. PLoS Biol. 2, e169 (2004).

4. Moser, M., Legate, K. R., Zent, R. \& Fassler, R. The tail of integrins, talin, and kindlins. Science 324, 895-899 (2009).

5. Calderwood, D. A., Campbell, I. D. \& Critchley, D. R. Talins and kindlins: partners in integrin-mediated adhesion. Nat. Rev. Mol. Cell Biol. 14, 503-517 (2013).

6. Arnaout, M. A. Biology and structure of leukocyte beta 2 integrins and their role in inflammation. F1000Res 5, 2433 (2016).

7. Goksoy, E. et al. Structural basis for the autoinhibition of talin in regulating integrin activation. Mol. Cell 31, 124-133 (2008).

8. Song, X. et al. A novel membrane-dependent on/off switch mechanism of talin FERM domain at sites of cell adhesion. Cell Res. 22, 1533-1545 (2012).

9. Goult, B. T. et al. The structure of an interdomain complex that regulates talin activity. J. Biol. Chem. 284, 15097-15106 (2009).

10. Goult, B. T. et al. Structural studies on full-length talin1 reveal a compact autoinhibited dimer: implications for talin activation. J. Struct. Biol. 184, 21-32 (2013).

11. Martel, V. et al. Conformation, localization, and integrin binding of talin depend on its interaction with phosphoinositides. J. Biol. Chem. 276, 21217-21227 (2001)

12. Yang, J. et al. Conformational activation of talin by RIAM triggers integrinmediated cell adhesion. Nat. Commun. 5, 5880 (2014).

13. Schiemer, J. et al. Galpha13 switch region 2 relieves talin autoinhibition to activate alphaIIbbeta3 integrin. J. Biol. Chem. 291, 26598-26612 (2016).

14. Vinogradova, O. et al. A structural mechanism of integrin alpha(IIb)beta(3) "inside-out" activation as regulated by its cytoplasmic face. Cell 110, 587-597 (2002).

15. Kim, M., Carman, C. V. \& Springer, T. A. Bidirectional transmembrane signaling by cytoplasmic domain separation in integrins. Science 301, 1720-1725 (2003)

16. Wegener, K. L. et al. Structural basis of integrin activation by talin. Cell 128, 171-182 (2007)

17. Xu, X. P. et al. Three-dimensional structures of full-length, membraneembedded human alpha(Ilb)beta(3) integrin complexes. Biophys. J. 110, 798-809 (2016).

18. Shattil, S. J., Kim, C. \& Ginsberg, M. H. The final steps of integrin activation: the end game. Nat. Rev. Mol. Cell Biol. 11, 288-300 (2010).

19. Zeiler, M., Moser, M. \& Mann, M. Copy number analysis of the murine platelet proteome spanning the complete abundance range. Mol. Cell Proteomics 13, 3435-3445 (2014).
20. Stritt, S. et al. Rap1-GTP-interacting adaptor molecule (RIAM) is dispensable for platelet integrin activation and function in mice. Blood 125, 219-222 (2015).

21. Klapproth, S. et al. Loss of the Rap1 effector RIAM results in leukocyte adhesion deficiency due to impaired beta2 integrin function in mice. Blood 126, 2704-2712 (2015).

22. $\mathrm{Su}, \mathrm{W}$. et al. Rapl and its effector RIAM are required for lymphocyte trafficking. Blood 126, 2695-2703 (2015).

23. Chrzanowska-Wodnicka, M., Smyth, S. S., Schoenwaelder, S. M., Fischer, T. H. \& White, G. C. 2nd Rap1b is required for normal platelet function and hemostasis in mice. J. Clin. Invest. 115, 680-687 (2005).

24. Nieswandt, B. et al. Loss of talin1 in platelets abrogates integrin activation, platelet aggregation, and thrombus formation in vitro and in vivo. J. Exp. Med. 204, 3113-3118 (2007).

25. Petrich, B. G. et al. Talin is required for integrin-mediated platelet function in hemostasis and thrombosis. J. Exp. Med. 204, 3103-3111 (2007).

26. Goult, B. T. et al. Structure of a double ubiquitin-like domain in the talin head: a role in integrin activation. EMBO J. 29, 1069-1080 (2010).

27. Plak, K., Pots, H., Van Haastert, P. J. \& Kortholt, A. Direct interaction between TalinB and Rap1 is necessary for adhesion of dictyostelium cells. BMC Cell Biol. 17, 1 (2016)

28. White, G. C. 2nd, Crawford, N. \& Fischer, T. H. Cytoskeletal interactions of Rap1b in platelets. Adv. Exp. Med. Biol. 344, 187-194 (1993).

29. Hancock, J. F. Ras proteins: different signals from different locations. Nat. Rev Mol. Cel. Biol. 4, 373-384 (2003).

30. Gloerich, M. \& Bos, J. L. Regulating rap small G-proteins in time and space. Trends Cell Biol. 21, 615-623 (2011).

31. Chrzanowska-Wodnicka, M. Rap1 in endothelial biology. Curr Opin Hematol. 24, 248-255 (2017).

32. Vaynberg, J. \& Qin, J. Weak protein-protein interactions as probed by NMR spectroscopy. Trends Biotechnol. 24, 22-27 (2006).

33. Perera, H. D. et al. Membrane binding of the $\mathrm{N}$-terminal ubiquitin-like domain of kindlin-2 is crucial for its regulation of integrin activation. Structure 19, 1664-1671 (2011).

34. Li, X. et al. Structural basis for small G protein effector interaction of Rasrelated protein 1 (Rap1) and adaptor protein Krev interaction trapped 1 (KRIT1). J. Biol. Chem. 287, 22317-22327 (2012).

35. Wynne, J. P. et al. Rap1-interacting adapter molecule (RIAM) associates with the plasma membrane via a proximity detector. J. Cell Biol. 199, 317-330 (2012).

36. Das, M., Subbayya Ithychanda, S., Qin, J. \& Plow, E. F. Mechanisms of talindependent integrin signaling and crosstalk. Biochim. Biophys. Acta 1838, 579-588 (2014).

37. Bouaouina, M., Lad, Y. \& Calderwood, D. A. The N-terminal domains of talin cooperate with the phosphotyrosine binding-like domain to activate betal and beta3 integrins. J. Biol. Chem. 283, 6118-6125 (2008).

38. Theodosiou, M. et al. Kindlin-2 cooperates with talin to activate integrins and induces cell spreading by directly binding paxillin. Elife 5, e10130 (2016).

39. Pollard, T. D., Blanchoin, L. \& Mullins, R. D. Molecular mechanisms controlling actin filament dynamics in nonmuscle cells. Annu. Rev. Biophys. Biomol. Struct. 29, 545-576 (2000).

40. Elliott, P. R. et al. The Structure of the talin head reveals a novel extended conformation of the FERM domain. Structure 18, 1289-1299 (2010).

41. Gureasko, J. et al. Membrane-dependent signal integration by the Ras activator Son of sevenless. Nat. Struct. Mol. Biol. 15, 452-461 (2008).

42. Pizon, V., Lerosey, I., Chardin, P. \& Tavitian, A. Nucleotide sequence of a human cDNA encoding a ras-related protein (rap1B). Nucleic Acids Res. 16, 7719 (1988).

43. Lilja, J. et al. SHANK proteins limit integrin activation by directly interacting with Rap1 and R-Ras. Nat. Cell Biol. 19, 292-305 (2017).

44. Lagarrigue, F. et al. A RIAM/lamellipodin-talin-integrin complex forms the tip of sticky fingers that guide cell migration. Nat. Commun. 6, 8492 (2015).

45. Gavin, A. C. et al. Functional organization of the yeast proteome by systematic analysis of protein complexes. Nature 415, 141-147 (2002).

46. Hein, M. Y. et al. A human interactome in three quantitative dimensions organized by stoichiometries and abundances. Cell 163, 712-723 (2015).

47. Williamson, M. P. Using chemical shift perturbation to characterise ligand binding. Prog. Nucl. Magn. Reson. Spectrosc. 73, 1-16 (2013).

48. Xu, Y., Wang, X., Yang, J., Vaynberg, J. \& Qin, J. PASA--a program for automated protein NMR backbone signal assignment by pattern-filtering approach. J. Biomol. NMR 34, 41-56 (2006).

49. Delaglio, F. et al. NMRPipe: a multidimensional spectral processing system based on UNIX pipes. J. Biomol. NMR 6, 277-293 (1995).

50. Garrett, D. S., Powers, R., Gronenborn, A. M. \& Clore, G. M. A common sense approach to peak picking in two-, three-, and four-dimensional spectra using automatic computer analysis of contour diagrams. 1991. J. Magn. Reson. 213, 357-363 (2011) 
51. Goddard T. D. \& Kneller D. G. SPARKY 3 (University of California, San Francisco). http://www.cgl.ucsf.edu/home/sparky/.

52. Schwieters, C. D., Kuszewski, J. J., Tjandra, N. \& Clore, G. M. The Xplor-NIH NMR molecular structure determination package. J. Magn. Reson. 160, 65-73 (2003).

53. Shen, Y., Delaglio, F., Cornilescu, G. \& Bax, A. TALOS+: a hybrid method for predicting protein backbone torsion angles from NMR chemical shifts. $J$ Biomol. NMR 44, 213-223 (2009).

54. Laskowski, R. A., Rullmannn, J. A., MacArthur, M. W., Kaptein, R. \& Thornton, J. M. AQUA and PROCHECK-NMR: programs for checking the quality of protein structures solved by NMR. J. Biomol. NMR 8, 477-486 (1996).

55. Moore, D. T. et al. Affinity of talin-1 for the beta3-integrin cytosolic domain is modulated by its phospholipid bilayer environment. Proc. Natl Acad. Sci. USA 109, 793-798 (2012).

56. Austen, K. et al. Extracellular rigidity sensing by talin isoform-specific mechanical linkages. Nat. Cell Biol. 17, 1597-1606 (2015).

57. Austen, K., Kluger, C., Freikamp, A., Chrostek-Grashoff, A. \& Grashoff, C. Generation and analysis of biosensors to measure mechanical forces within cells. Methods Mol. Biol. 1066, 169-184 (2013).

\section{Acknowledgements}

We thank Reinhard Fässler for providing us with talin $1 / 2$ double-knockout fibroblasts. This work was supported by NIH grants R01HL58758, R01GM62823 to J.Q., P01HL073311 to E.F.P., the Max-Planck-Society, and the SFB1123 TP A08 to M.M. We thank Jianmin Liu, Koichi Fukuda, Sujay Ithychanda, and Xi-An Mao for assistance and useful discussions.

\section{Author contributions}

L.Z., M.M. and J.Q. conceived this study. L.Z. performed all biochemical/biophysical studies including NMR 2D-HSQC experiments, GST pull-down assays, ITC experiments, and MST assay. L.Z. and J.Y. performed all 3D-NMR studies and determined the solution structure of Raplb/talin-F0 complex. T.B. and S.K. performed the functional experiments showing that the double mutations of talin resulted in defective cell adhesion and spreading, and T.B., S.K. and M.M. analyzed the data. A.H. and J.H. performed the integrin activation assay in CHO A5 cells. L.Z., F.L. and J.Y. designed and performed vesicle co-sedimentation assays. L.Z. and H.L. prepared various types of cell lysates and analyzed the expression levels of desired proteins. L.Z. and K.S. prepared ${ }^{15} \mathrm{~N} /{ }^{13} \mathrm{C}$-labeled Rap1 and ${ }^{15} \mathrm{~N} /{ }^{13} \mathrm{C}$-labeled talin-F0 for 3D-NMR studies. T.B. and E.F.P. participated in the interpretation and preparation of the manuscript. L.Z., M.M. and J.Q. wrote the manuscript with contributions from all other co-authors.

\section{Additional information}

Supplementary Information accompanies this paper at doi:10.1038/s41467-017-01822-8

Competing interests: The authors declare no competing financial interests.

Reprints and permission information is available online at http://npg.nature.com/ reprintsandpermissions/

Publisher's note: Springer Nature remains neutral with regard to jurisdictional claims in published maps and institutional affiliations.

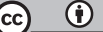

Open Access This article is licensed under a Creative Commons Attribution 4.0 International License, which permits use, sharing, adaptation, distribution and reproduction in any medium or format, as long as you give appropriate credit to the original author(s) and the source, provide a link to the Creative Commons license, and indicate if changes were made. The images or other third party material in this article are included in the article's Creative Commons license, unless indicated otherwise in a credit line to the material. If material is not included in the article's Creative Commons license and your intended use is not permitted by statutory regulation or exceeds the permitted use, you will need to obtain permission directly from the copyright holder. To view a copy of this license, visit http://creativecommons.org/ licenses/by/4.0/.

(C) The Author(s) 2017 